ARTICLE

\title{
Global kinome profiling reveals DYRK1A as critical activator of the human mitochondrial import machinery
}

Corvin Walter 1,2,12, Adinarayana Marada1,12, Tamara Suhm¹, Ralf Ernsberger', Vera Muders', Cansu Kücükköse ${ }^{1,2}$, Pablo Sánchez-Martín', Zehan Hu (1) ${ }^{3}$, Abhishek Aich (1) ${ }^{4,5}$, Stefan Loroch ${ }^{6}$, Fiorella Andrea Solari ${ }^{6}$, Daniel Poveda-Huertes (10 1, Alexandra Schwierzok', Henrike Pommerening ${ }^{1}$, Stanka Matic ${ }^{1}$, Jan Brix ${ }^{1}$, Albert Sickmann (1) ${ }^{6}$, Claudine Kraft (1) ${ }^{1,7}$, Jörn Dengjel (D) ${ }^{3}$, Sven Dennerlein ${ }^{4}$, Tilman Brummer (10) $8,9,10$, F.-Nora Vögtle ${ }^{1,7,11 凶} \&$ Chris Meisinger (1) $1,7,9 凶$

The translocase of the outer mitochondrial membrane TOM constitutes the organellar entry gate for nearly all precursor proteins synthesized on cytosolic ribosomes. Thus, TOM presents the ideal target to adjust the mitochondrial proteome upon changing cellular demands. Here, we identify that the import receptor TOM70 is targeted by the kinase DYRK1A and that this modification plays a critical role in the activation of the carrier import pathway. Phosphorylation of TOM70 ${ }^{\text {Ser91 }}$ by DYRK1A stimulates interaction of TOM70 with the core TOM translocase. This enables transfer of receptor-bound precursors to the translocation pore and initiates their import. Consequently, loss of TOM70 ${ }^{\mathrm{Ser} 91}$ phosphorylation results in a strong decrease in import capacity of metabolite carriers. Inhibition of DYRK1A impairs mitochondrial structure and function and elicits a protective transcriptional response to maintain a functional import machinery. The DYRK1A-TOM70 axis will enable insights into disease mechanisms caused by dysfunctional DYRK1A, including autism spectrum disorder, microcephaly and Down syndrome.

\footnotetext{
${ }^{1}$ Institute of Biochemistry and Molecular Biology, ZBMZ, Faculty of Medicine, University of Freiburg, Freiburg, Germany. ${ }^{2}$ Faculty of Biology, University of Freiburg, Freiburg, Germany. ${ }^{3}$ Department of Biology, University of Fribourg, Fribourg, Switzerland. ${ }^{4}$ Department of Cellular Biochemistry, University Medical Center Göttingen, Göttingen, Germany. ${ }^{5}$ Cluster of Excellence "Multiscale Bioimaging: from Molecular Machines to Networks of Excitable Cells" (MBExC), University of Göttingen, Göttingen, Germany. ${ }^{6}$ Leibniz Institut für Analytische Wissenschaften - ISAS - e.V., Dortmund, Germany. ${ }^{7}$ CIBSS - Centre for Integrative Biological Signalling Studies, University of Freiburg, Freiburg, Germany. ${ }^{8}$ Institute of Molecular Medicine, ZBMZ, Faculty of Medicine, University of Freiburg, Freiburg, Germany. ${ }^{9}$ BIOSS Centre for Biological Signalling Studies, University of Freiburg, Freiburg, Germany.

${ }^{10}$ German Cancer Consortium DKTK Partner Site Freiburg, German Cancer Research Center (DKFZ), Heidelberg, Germany. ${ }^{11}$ Present address: Center for Molecular Biology of Heidelberg University (ZMBH), Heidelberg, Germany. ${ }^{12}$ These authors contributed equally: Corvin Walter, Adinarayana Marada.

凶email: n.voegtle@zmbh.uni-heidelberg.de; chris.meisinger@biochemie.uni-freiburg.de
} 
$\mathrm{M}$ itochondria are essential organelles in eukaryotic cells and play numerous functions important e.g. for energy supply, biosynthesis of metabolites, and cofactors or regulation of programmed cell death. All these functions rely on an extensive and functional organellar proteome, which is mainly built by import of nuclear-encoded precursor proteins from the cytosol $^{1-6}$. Dedicated signal sequences direct the cytosolic precursors to the translocase of the outer membrane (TOM), from where they are sorted to their suborganellar destinations ${ }^{7-13}$. The two most comprehensive subproteomes of mitochondria ${ }^{14}$, the inner membrane and the matrix are built via two main import routes, termed the carrier- and presequence-import pathway. Metabolite carriers are polytopic inner membrane proteins and possess internal, non-cleavable targeting signals. They are typically delivered by cytosolic chaperones to the import receptor Tom70 and then translocated via TOM into the intermembrane space ${ }^{15-21}$. The TIM22 translocase then mediates sorting and assembly in the inner membrane ${ }^{22-25}$. Presequences are Nterminal extensions that serve as targeting signals for import into the matrix and are mainly recognized and bound by the import receptors Tom 20 and Tom 22 . After translocation across the outer membrane the presequence precursors are handed over to the TIM23 complex from where they are imported into the matrix or, in case of single spanning membrane proteins laterally sorted into the inner membrane ${ }^{14,26-28}$.

Recent studies revealed that the main import receptors Tom 20 and Tom70 are rather loosely associated with the core TOM complex, which consists of Tom40, Tom22, and the small Tom proteins Tom5, 6, and 7 19,21,29-32. While the mitochondrial import translocases have been considered for long time static and constitutively active entities, studies in the model organism $S$. cerevisiae have shown that components of the organellar protein entry gate TOM are modified by cytosolic protein kinases and allow regulation of the import flux upon changing cellular conditions ${ }^{33-35}$. However, if such regulatory mechanisms exist for the human mitochondrial import machinery remains enigmatic. The human kinome comprises 550 different kinases and thus exceeds the yeast kinome by more than 5 -fold ${ }^{36}$. This large number of kinases makes the discovery of signaling pathways that regulate mitochondrial protein biogenesis in humans challenging.

Here, we performed a global screen for kinase candidates of the mitochondrial import receptor TOM70 and identified DYRK1A as critical activator of the human import machinery. Interestingly, dysfunctional DYRK1A was described to cause an array of disease phenotypes including intellectual disability, speech delay, motor deficits, autism, and microcephaly (DYRK1A-related syndrome). Moreover, DYRK1A is localized in the Down syndrome critical region of chromosome 21 and is considered a strong candidate for learning defects associated with Down syndrome ${ }^{37-42}$. Several of these clinical manifestations of DYRK1A-related syndrome, particularly in their combinations, suspiciously resemble phenotypes described of mitochondrial diseases ${ }^{18,43-45}$. This led us to speculate that dysfunctional DYRK1A signaling affects mitochondrial integrity and that this is mediated by impaired protein import.

We found that the import receptor TOM70 is almost quantitatively phosphorylated in human cells in vivo and that DYRK1A exclusively targets residue Serine 91. Furthermore, phosphorylation of TOM70 is crucial for activation of the metabolite carrier import pathway. We analyzed the impact of TOM70 ${ }^{\text {Ser91 }}$ phosphorylation on mitochondrial protein biogenesis on the molecular level and found that DYRK1A phosphorylation at $\mathrm{TOM} 70^{\mathrm{Ser} 91}$ does not affect binding of precursor proteins, but strongly stimulates the interaction of the import receptor with the core TOM machinery. Consequently, selective inhibition of DYRK1A signaling in vivo leads to impairment of crucial mitochondrial functions, which is mitigated by a protective transcriptional response and remodeling of the import machinery. Our findings reveal an unexpected link of mitochondrial protein biogenesis and DYRK1A signaling and can enable an understanding of the pathophysiological consequences of dysfunctional DYRK1A in DYRK1A-related syndrome, Autism Spectrum Disorder, and Down syndrome.

\section{Results}

DYRK1A targets the mitochondrial import receptor TOM70. To search for kinase candidates targeting the human mitochondrial import machinery we employed a KinaseFinder platform which is based on a radiometric filter binding assay ${ }^{46}$ using ${ }^{33} \mathrm{P}$ ATP (ProQinase (Freiburg, Germany)) and allows to test for 339 different human protein kinases experimentally in an unbiased approach. The soluble cytosolic domain of human TOM70 $\left(\mathrm{TOM} 70_{\mathrm{cd}}\right)$, which represents the main import receptor for the carrier import pathway, was used as substrate (Supplementary Fig. 1a). The two DYRK1 isoforms DYRK1A and DYRK1B showed the by far highest scores in phosphorylating TOM $70_{c d}$ (Fig. 1a). Furthermore, the kinase activities of both, DYRK1A and DYRK1B, were dependent on the amount of added TOM70 protein (Supplementary Fig. 1b).

To validate these results further we incubated TOM70 $\mathrm{cd}$ in the presence or absence of DYRK1A and monitored phosphorylation activity via phosphate affinity (Phos-tag) SDS-PAGE that retards the gel mobility of phosphorylated proteins $33,34,47,48$. TOM70 $0_{\mathrm{cd}}$ migrated slower upon incubation with DYRK1A (Fig. 1b, lanes 5 and 6), indicating a DYRK1A-dependent phosphorylation of TOM $70_{\mathrm{cd}}$. In contrast, the receptor domains of TOM $22_{\mathrm{cd}}$ and TOM $20_{c d}$ did not show a changed migration upon incubation with DYRK1A indicating that DYRK1A and DYRK1B exclusively target the TOM70 receptor (Fig. 1b, lanes 1-4 and Supplementary Fig. 1a).

Notably, DYRK1A and DYRK1B have not been linked to mitochondria before. DYRK1A and DYRK1B are two isoforms with almost identical catalytic sites ${ }^{49}$. DYRK1A is ubiquitously expressed, located in the nucleus and cytosol and involved in many different cellular processes including cell differentiation, proliferation, and survival ${ }^{49-52}$. The isoform DYRK1B has similar functions as DYRK1A, but is only expressed in a very limited spectrum of cell types ${ }^{49}$. Thus, we focused on a possible link between DYRK1A and mitochondria and assessed its potential role in regulating the human mitochondrial protein import machinery.

To identify which residues of TOM70 are targeted by DYRK1A, we profiled phosphorylation sites in isolated mitochondria from HEK293T cells by phosphoproteomics. Phosphopeptide enrichment using ERLIC followed by LC-MS/MS analysis revealed three phosphorylated residues on TOM70: Thr85, Ser91, and Ser110 (Supplementary Fig. 1c and Methods section). To test which of these residues might represent targets of DYRK1A, we purified variants of the TOM70 receptor domain harboring alanine residues that cannot be phosphorylated at the respective positions (Supplementary Fig. 1d). In vitro phosphorylation of these domains by DYRK1A followed by Phos-tag gel electrophoresis revealed that the wild-type (WT) TOM70, TOM70 ${ }^{\mathrm{T} 85 \mathrm{~A}}$, and TOM70 $5110 \mathrm{~A}$ but not the TOM70 $591 \mathrm{~A}$ domains were phosphorylated by DYRK1A (Fig. 1c). This indicates that DYRK1A can phosphorylate human TOM70 at residue Ser91. While these in vitro assays revealed Ser91 as target of DYRK1A we wondered about the abundance and relevance of this phosphorylation site in vivo. For this, we analyzed mitochondria isolated from HEK293T cells by two approaches. Firstly, Phos-tag gel electrophoresis of non-treated mitochondria revealed a 

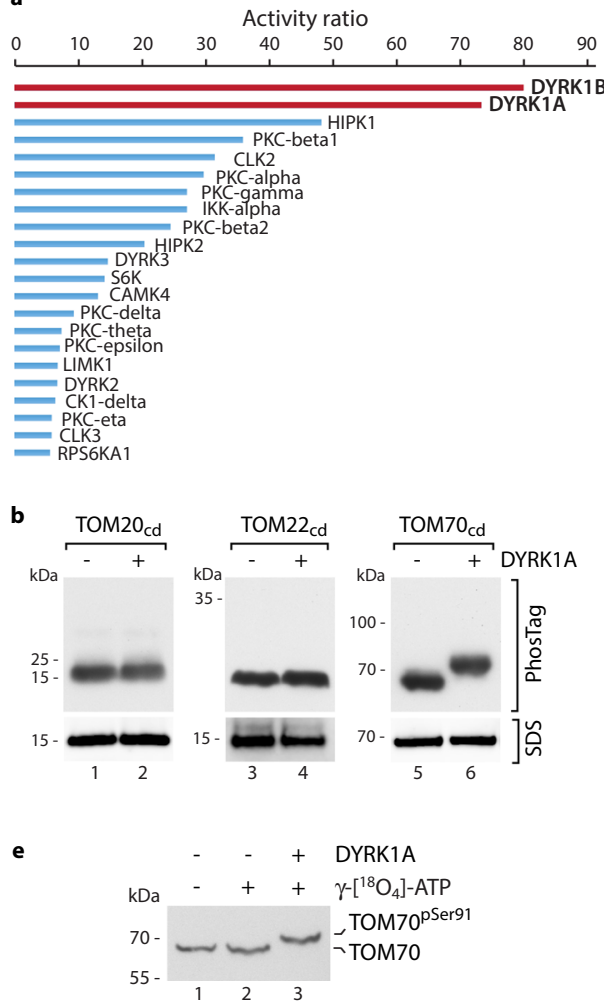

c

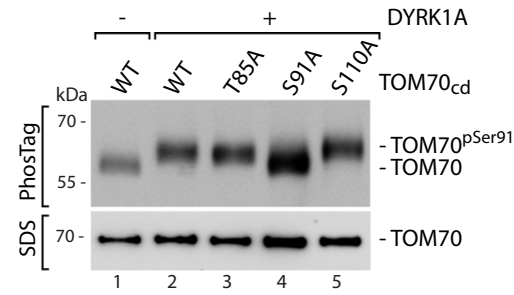

d
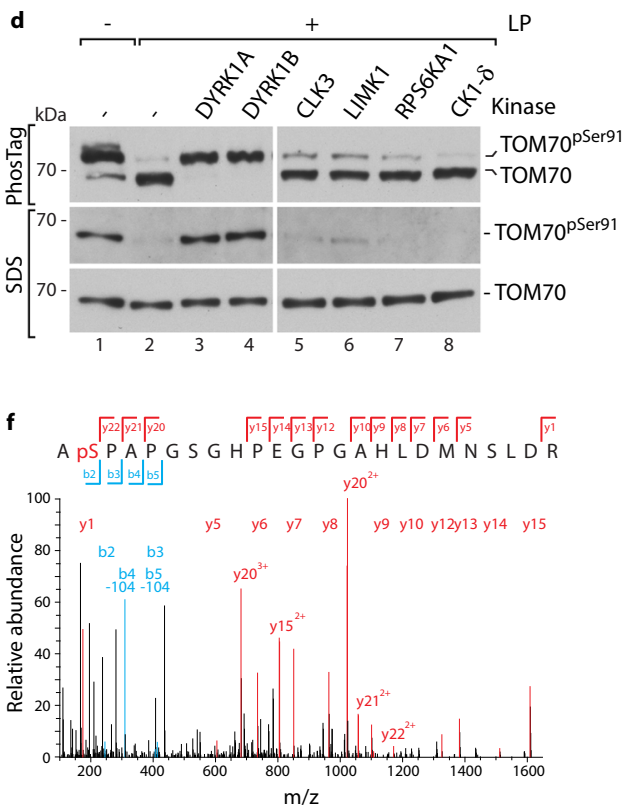

Fig. 1 Identification of DYRK1A as kinase of the mitochondrial import receptor TOM70. a Results of KinaseFinder assay (ProQinase ${ }^{T M}$ ) for human $\mathrm{TOM}_{\mathrm{Cd}}$ tested for 339 different protein kinases. Shown are activity ratios of $33 \mathrm{P}$-ATP-radiometric filter binding assays. $\mathbf{b}$ In vitro phosphorylation assay of soluble cytosolic domains (cd) of the human import receptors TOM70, TOM20, and TOM22. Proteins were incubated in the presence or absence of human DYRK1A. Samples were analyzed via Phos-tag electrophoresis, SDS-PAGE and immunoblotting using the respective TOM antibody (Supplementary Table 1). c In vitro kinase assay of indicated $T O M 70_{c d}$ variants in the absence or presence of DYRK1A. Samples were analyzed by Phos-tag gel electrophoresis (upper panel) or standard SDS-PAGE (lower panel) followed by immunoblotting using TOM70 antisera. d Analysis of isolated mitochondria from HEK293T cells incubated in the presence or absence of lambda phosphatase (LP). Where indicated samples were incubated with the respective kinase after LP treatment. Samples were analyzed by Phos-tag gels (upper panel) or standard SDS gels (middle and lower panel). Upper and lower panels show immunoblots stained with TOM70 antibody and middle panel was stained with TOM70Ser91 phosphospecific antibody. e Isolated mitochondria were incubated with $\gamma-\left[{ }^{18} \mathrm{O}_{4}\right]$-ATP and DYRK1A where indicated. Samples were analyzed via Phos-tag gels and immunoblotting using TOM70 antibody. $\mathbf{f ~ M S / M S ~ s p e c t r u m ~ f r o m ~ m i t o c h o n d r i a ~ i n c u b a t e d ~ w i t h ~} \gamma-\left[{ }^{18} \mathrm{O}_{4}\right]$-ATP shown in e localizing the DYRK1A-dependent phosphorylation of TOM70 to Ser91. Identified b- and y-ions are listed in blue and red, respectively. The b2-y22 ion pair localizes the phosphorylation unambiguously to Ser91. The amino acid sequence of the respective tryptic peptide TOM7090-113 is indicated. Unprocessed immunoblots are reported in the Source Data File.

prominent TOM70 immunoreactive band that was accessible to treatment with lambda phosphatase (LP) (Fig. 1d, upper panel, lanes 1 and 2). This indicates that TOM70 is almost quantitatively phosphorylated in vivo. Secondly, analysis of the same samples via SDS-PAGE and immunodecoration with phospho-Ser91 specific TOM70 antiserum revealed loss of the signal upon LP treatment while the general TOM70 immunoreactivity was not affected (Fig. 1d, middle and lower panel, lanes 1 and 2 and Supplementary Fig. 1e). Notably, in both approaches, addition of DYRK1A or DYRK1B kinase after phosphatase (LP) treatment resulted in redetection of the Ser91 phosphorylation (Fig. 1d, lanes 3 and 4) while kinases with low scores in our kinase screen (Fig. 1a) revealed no significant changes (Fig. 1d, lanes 5-8 and Supplementary Fig. 1f, g). Interestingly, HIPK1 and CLK2, members of the DYRK kinase family and the closely related CLK family ${ }^{51}$, respectively, which scored in a medium-range in the Kinase Finder screen, were also able to phosphorylate TOM70 Ser91, however, to a lesser extent compared to DYRK1A and DYRK1B (Supplementary Fig. 1h). To analyze if further subunits of the TOM import machinery are targeted by DYRK1A we incubated isolated and phosphatase (LP) treated mitochondria in the presence or absence of DYRK1A and included $\gamma$ - $\left[{ }^{18} \mathrm{O}_{4}\right]$-ATP to detect phosphorylated residues directly dependent on the presence of DYRK1A by phosphoproteomics (Fig. 1e). LC-MS/ MS analysis revealed residue Ser91 of TOM70 as the only site of the entire import machinery that was specifically phosphorylated by DYRK1A (Fig. If and Methods section).

In summary, our data show that human TOM70 is nearly quantitatively phosphorylated at Ser91 in vivo and that this position is specifically targeted by DYRK1A in vitro and in isolated mitochondria.

DYRK1A signaling is required for functional mitochondria in vivo. To investigate if DYRK1A also targets TOM70 in vivo and if its phosphorylation of TOM70 impacts on mitochondrial protein biogenesis (e.g. displaying an activatory or inhibitory role) we treated cells with INDY, a benzothiazole derivative that was developed as potent and selective DYRK1A inhibitor ${ }^{53}$ and can indeed impair TOM70 Ser91 phosphorylation by DYRK1A 
in vitro (Supplementary Fig. 2a). Phos-tag gel electrophoresis of cells incubated overnight in the presence or absence of INDY revealed a significant change in the ratio of phosphorylated to non-phosphorylated TOM70 indicating that DYRK1A targets TOM70 in vivo (Supplementary Fig. 2b). Next, we aimed to analyze how mitochondrial functions would be affected upon compromised DYRK1A activity. At first, respiratory activity in the presence or absence of INDY was tested by real-time respirometry and revealed a significantly impaired function of the OXPHOS machinery upon DYRK1A inhibition (Fig. 2a). We also found a strong decrease in cell growth upon DYRK1A inhibition (Supplementary Fig. 2c). When we analyzed mitochondrial morphology by fluorescence microscopy, we observed a severe loss of the typical elongated, tubular mitochondrial structure in INDYtreated cells (Fig. 2b, c). Moreover, the INDY-induced aberrant mitochondrial morphology seemed to be in part mediated by the TOM70 Ser91 phosphorylation as overexpression of TOM70 WT but not of the non-phosphorylatable TOM70 ${ }^{S 91 \mathrm{~A}}$ variant was able to rescue this phenotype (Fig. 2b, c and Supplementary Fig. 2d).

Taken together, DYRK1A activity is required for phosphorylation of TOM70 at position Ser91 in vivo. Disturbed DYRK1A signaling leads to impairment of mitochondrial structure, reduced respiration rates, and retardation of cell growth. Thus, TOM70 Ser91 phosphorylation by DYRK1A appears to exert an activating role in mitochondrial protein biogenesis required to maintain mitochondrial function.

DYRK1A inhibition elicits a protective transcriptional response and remodeling of the import machinery. We next aimed to analyze if INDY-mediated inhibition of DYRK1A would indeed cause an impairment of TOM70-dependent protein biogenesis. We tested this by importing radiolabelled TIM 23 precursor, a model substrate of the TOM70-dependent carrier import pathway $24,25,54$, into mitochondria that were isolated from cells grown in the presence or absence of INDY. Surprisingly, import of TIM23, monitored via Blue Native PAGE, was only mildly affected (Fig. 3a). When analyzing the TOM70 phosphorylation status, we noticed that INDY treatment had changed the ratio of phosphorylated towards non-phosphorylated TOM70, but had also led to an overall increase in TOM70 protein levels. This increase in TOM70 abundance resulted in similar amounts of phosphorylated TOM70 species in INDY-treated and in non-treated cells (Supplementary Fig. 2b). This maintained level in TOM70 Ser91 phosphorylation would explain why the carrier import pathway was still relatively functional upon INDY treatment (Fig. 3a). When we further analyzed protein levels via standard SDS-PAGE and immunoblotting, we noticed that next to TOM70 also the other two TOM import receptors, TOM22 and TOM20 were increasing upon DYRK1A inhibition, whereas the central translocation pore TOM40 was not affected (Fig. 3b). Therefore, we speculated that DYRK1A inhibition might trigger a transcriptional response that leads to upregulation of TOMM70 and possibly also other import receptors to mitigate TOM70 impairment. We tested this by qRT-PCR analysis and observed that INDY treatment led to a significant increase in transcript levels of TOMM70 and TOMM20 (Fig. 3c). Furthermore, we found that also the transcripts of $D Y R K 1 A$, its isoform $D Y R K 1 B$, and further kinases of the DYRK family (that had been identified with lower scores in our KinaseFinder assay (Fig. 1a and Supplementary Fig. 1h)) significantly increased (Supplementary Fig. 3). To expose the impact of TOM70 pS91 on the carrier import pathway, we treated cells with INDY while in parallel inducing TOMM70 downregulation via shRNA (see Methods section) to counteract the transcriptional response. With this experimental setup we could now indeed detect decreased levels of phosphorylated TOM70 upon INDY treatment and as a consequence a strong impairment a

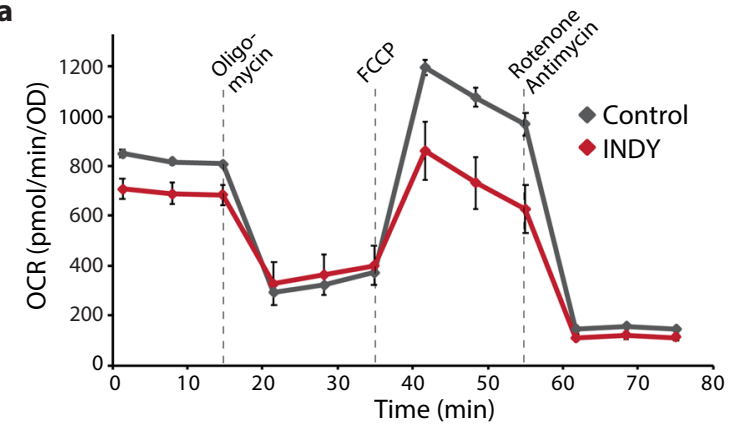

b
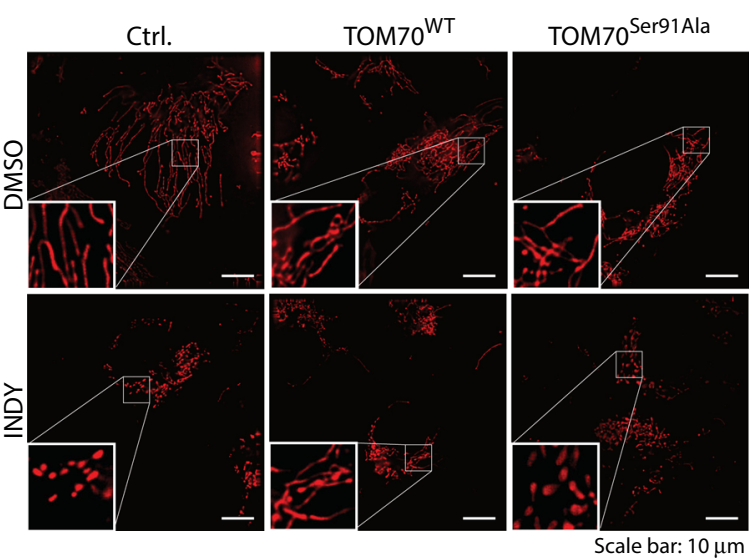

C
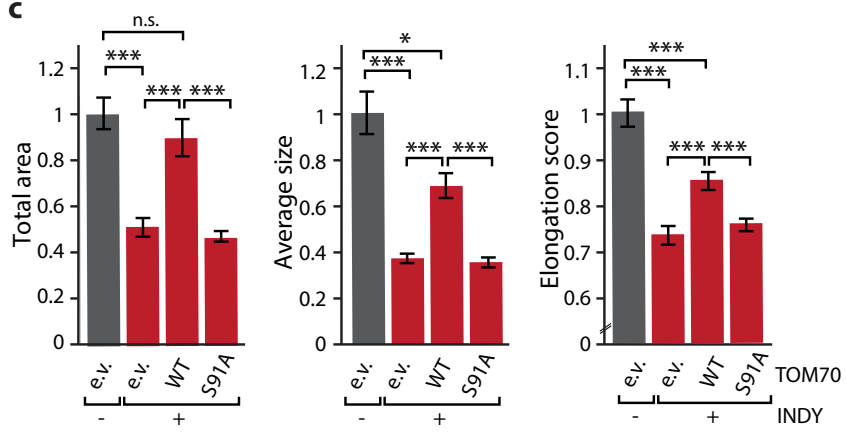

Fig. 2 Inhibition of DYRK1A activity in vivo affects function and morphology of mitochondria. a Analysis of oxygen consumption rates (OCR) of cells treated with INDY $(10 \mu \mathrm{M})$ at basal conditions and after addition of indicated compounds. Control cells were incubated in parallel in the presence of the respective volume of DMSO. Data represent means \pm SEM from six independent experiments. b U2OS cells cultured on glass bottom plates were treated with $10 \mu \mathrm{M}$ INDY or DMSO as control for $24 \mathrm{~h}$ and stained with MitoTracker Red CMXRos. Where indicated cells were transfected with TOM70WT and TOM70 Ser91Ala overexpression plasmids. Each microscopy image includes a magnified inset. Scale bar: $10 \mu \mathrm{m}$. c Total mitochondrial area, average size and elongation of the samples from $\mathbf{b}$ were measured. Data were normalized to the respective DMSO control and shown as means \pm SEM ( $n=20$ cells). Statistical analysis was performed using a one-way ANOVA followed by a Bonferroni post-hoc test to allow multiple comparisons. Significances are indicated with asterisks: ${ }^{\star \star \star} p<$ $0.001,{ }^{\star \star} p<0.01,{ }^{\star} p<0.05$, not significant (n.s.) $p>0.05$. Numerical source data are reported in the Source Data File.

of the carrier import pathway was revealed (Fig. 3d, e). Notably, mitochondria isolated from DYRK1A knock-out cells revealed reduced levels of TOM70 pS91 and consequently also showed compromised carrier import (Supplementary Fig. 4).

We conclude that inhibition of DYRK1A signaling causes a protective transcriptional response that includes upregulation of 
a

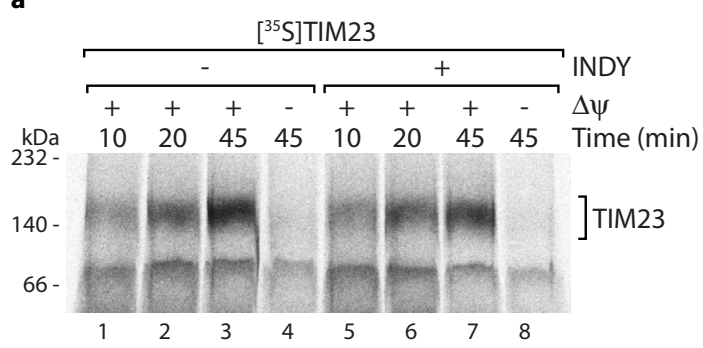

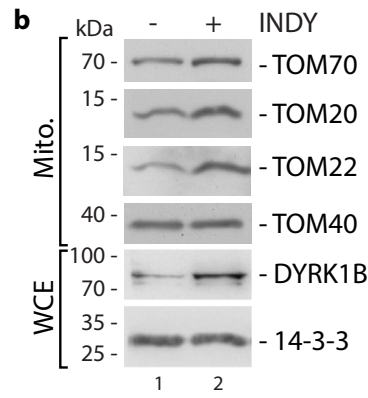
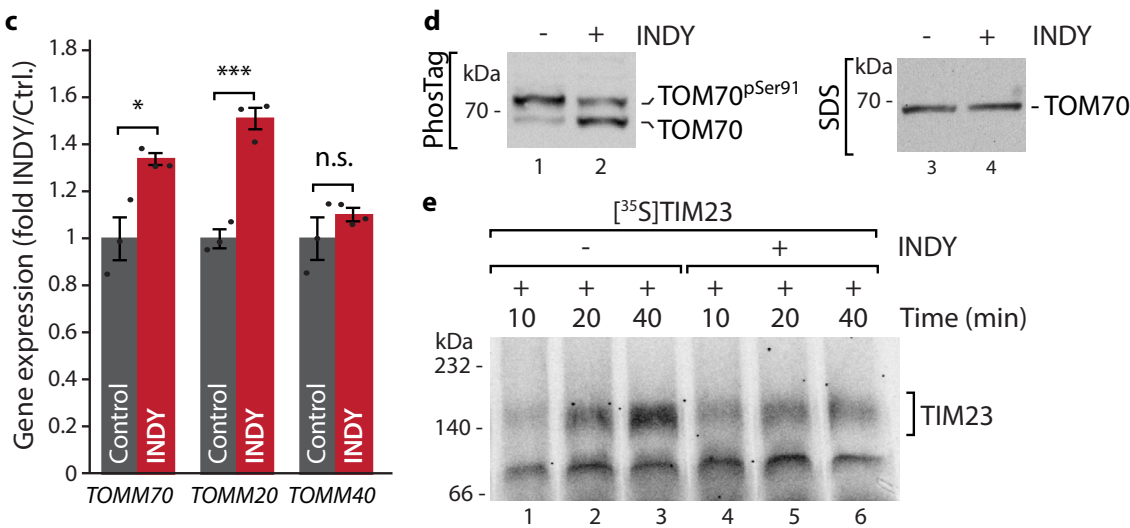

Fig. 3 DYRK1A inhibition elicits a protective transcriptional response resulting in remodeling of the import machinery. a Import of radiolabelled TIM23 precursor into isolated mitochondria from cells grown overnight in the presence or absence of INDY $(10 \mu M)$. Where indicated the membrane potential $\Delta \psi$ was dissipated prior to the import reaction. Samples were lysed in digitonin buffer and subjected to Blue Native PAGE. Imported protein was analyzed by autoradiography. b Analysis of protein levels after INDY treatment (samples from a) of isolated mitochondria (Mito.) or whole cell exctracts (WCE) via standard SDS-PAGE and immunoblotting. 14-3-3, loading control. c Analysis of changes of indicated transcript levels upon DYRK1A inhibition (INDY treatment, $10 \mu \mathrm{M})$ by qRT-PCR. $n=3$, data represent mean \pm SEM. Statistical analysis was performed using a two-sided Student's $t$ test to compare between two groups ( $p=0.0132$ (TOMM70); $p=0.0007$ (TOMM20); $p=0.1734$ (TOMM40); n.s., not significant). Data are representative of two independent experiments. d TOM70 Phos-tag gels (lanes 1 and 2) and SDS-PAGE analysis (lanes 3 and 4) of mitochondria isolated from cells grown overnight in the presence or absence of INDY $(10 \mu \mathrm{M})$ and, for both conditions, in the presence of TOM70 shRNA. Samples were analyzed by immunodecoration using antisera against TOM70. e Import of radiolabelled TIM23 precursor into isolated mitochondria from cells grown overnight in the presence or absence of INDY $(10 \mu \mathrm{M})$ and in the presence of TOM70 shRNA (as in $\mathbf{d}$ ). Numerical source data and unprocessed immunoblots and autoradiography scans are reported in the Source Data File.

import receptors and further DYRK family members to compensate for loss of DYRK1A activity. This response is likely transient, as comparison of chemical inhibition of DYRK1A with a CRISPR-Cas9 generated DYRK1A knock-out also revealed compromised carrier import. Similarly, attenuation of the transcriptional response via TOMM70 shRNA also results in specific impairment of the carrier import pathway caused by compromised DYRK1A signaling.

TOM70 Ser91 phosphorylation facilitates interaction with the TOM core complex. To study how DYRK1A-dependent phosphorylation of TOM70 can influence mitochondrial protein biogenesis at a molecular level we first tested if Ser91 phosphorylation can modulate mitochondrial import of the TOM70 precursor itself. For this we generated radiolabelled precursor variants: TOM $70^{S 91 \mathrm{~A}}$, that cannot be phosphorylated and TOM $70^{S 91 E}$ mimicking the phosphorylated residue. Import into mitochondria was tested by two different assays. (i) Integration of TOM70 into the outer membrane by carbonate extraction ( $\mathrm{pH}$ 11.5) that separates integral membrane proteins from peripherally associated proteins and (ii) assembly of imported TOM70 by Blue Native PAGE. Analysis of the carbonate-resistant mitochondrial pellets after the import reaction revealed that all ${ }^{35} \mathrm{~S}$-labeled TOM70 variants integrated into the outer membrane in the same time-dependent manner (Fig. 4a). Analysis on Blue Native PAGE demonstrated that all variants assembled with similar efficiency in a complex at $\sim 200 \mathrm{kDa}$ probably reflecting formation of TOM70 dimers. Thus, the modification at Ser91 seems not to affect biogenesis of the TOM70 precursor itself (Fig. $4 \mathrm{a}-\mathrm{c}$ ). We next asked if DYRK1A activity might affect the precursor receptor function of TOM70, i.e. modulate its binding efficiency to precursor proteins. For this we employed a direct binding assay for precursor proteins using purified $\mathrm{TOM} 70_{\mathrm{cd}}$ immobilized via deca-His tag to Ni-NTA beads ${ }^{15,33} .{ }^{35}$ S-labeled precursor proteins are incubated with the TOM70 $\mathrm{cd}$ and, dependent on their binding affinity to the TOM70 receptor, specifically released from the beads together with TOM70 $\mathrm{cd}$ upon elution (Supplementary Fig. 5a). To assess the impact of phosphorylated Ser91, immobilized TOM70 $\mathrm{cd}$ was incubated in the absence or presence of DYRK1A prior to the addition of the radiolabelled precursor proteins. As model substrates of the carrier pathway we tested $\left[{ }^{35} \mathrm{~S}\right] \mathrm{TIM} 23$ and $\left[{ }^{35} \mathrm{~S}\right]$ ANT3 precursors $24,25,54$. Both precursors bound efficiently to the TOM70 receptor domain, however, their interaction was independent of previous phosphorylation of TOM $70_{c d}$ by DYRK1A (Fig. 4d and Supplementary Fig. 5b). $\left[{ }^{35} \mathrm{~S}\right] \mathrm{Hsp} 10$ as a model substrate of the presequence import pathway ${ }^{55,56}$ and $\left[{ }^{35}\right.$ S]GFP as a non-imported control protein were not binding to TOM $70_{c d}$ under any conditions (Fig. 4e, f and Supplementary Fig. 5c). Thus, phosphorylation of TOM70 ${ }^{\text {Ser91 }}$ does not seem to play a role in binding of metabolite carrier precursor proteins. Notably, Ser91 is located close to the transmembrane domain of TOM70 (Supplementary Fig. 5d) ${ }^{17,30}$. Therefore, we speculated that Ser91 


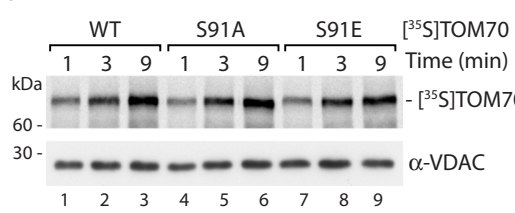

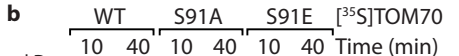

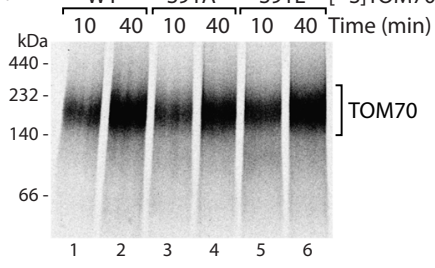

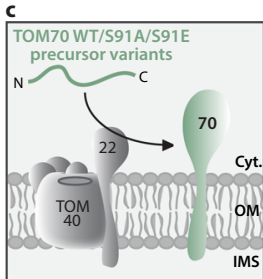

d

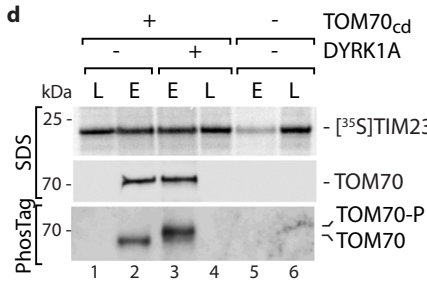

e

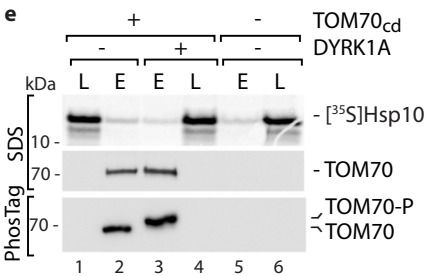

h

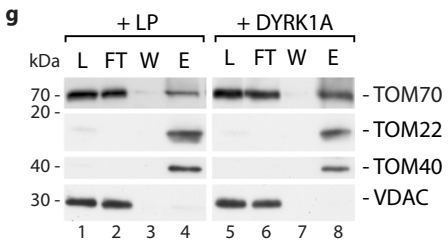

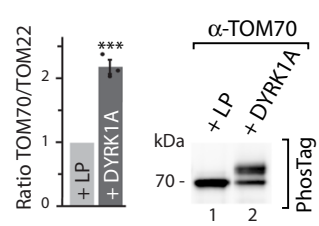
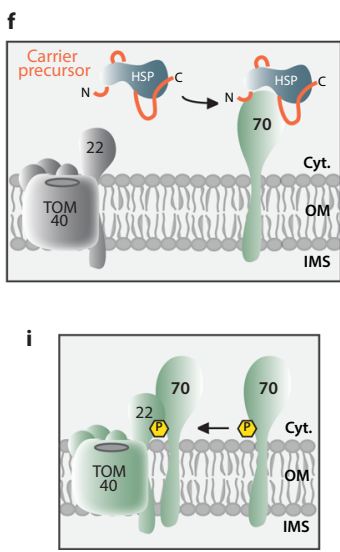

Fig. 4 Analysis of the role of TOM70Ser91 phosphorylation in the TOM complex. a Indicated $\left[{ }^{35} \mathrm{~S}\right] \mathrm{TOM} 70$ precursor variants were imported into isolated mitochondria for indicated time followed by protein extraction in $100 \mathrm{mM}$ sodiumcarbonate (pH 11.5). Carbonate-resistant pellet was loaded on SDS-PAGE and imported TOM70 was monitored by autoradiography after transfer onto PVDF membrane. VDAC immunostaining served as loading control. b Analysis of import reaction with indicated TOM70 variants via Blue Native PAGE followed by autoradiography. c Schematic overview of import assays performed in $\mathbf{a}$ and $\mathbf{b}$. d Binding assay for ${ }^{35}{ }^{3}$ ]TIM23 precursor (upper panel) to TOM70 ${ }_{\text {cd }}$ (immobilized via decaHis-tag on Ni-NTA beads) that was incubated before in the absence or presence of DYRK1A. TOM70 $c$ phosphorylation by DYRK1A was controlled via Phos-tag gels (lower panel). $L$ loaded radiolabelled precursor, E elution fraction containing released $\mathrm{TOM} \mathrm{O}_{\mathrm{cd}}$ and radiolabelled precursor in case of specific binding. e Binding of $\left[{ }^{35} \mathrm{~S}\right] \mathrm{Hsp} 10$ precursor to $\mathrm{TOM} \mathrm{C}_{\mathrm{cd}}$ pre-incubated with or without DYRK1A. Assay performed as in d. f Schematic overview of precursor binding assays performed in d and e. $\mathbf{g}$ Immunoprecipitation of TOM complex via TOM22 antibodies from mouse brain mitochondria that were incubated before in the presence of lambda phosphatase (LP; TOM70 dephosphorylated) or DYRK1A (TOM70 phosphorylated). Samples were analyzed by SDS-PAGE followed by immunoblotting with indicated antibodies. L load, FT flowthrough, W wash, E elution. h Quantification of TOM70 co-immunoprecipitated with the TOM core complex component TOM22 as shown in $\mathbf{g}$. Data represent mean \pm SEM from three independent experiments. Statistical analysis was performed using a two-sided Student's $t$ test to compare between two groups ( $p=0.0002$ ). Phos-tag analysis of TOM70 from samples used for immunoprecipitation in $\mathbf{g}$. i Schematic overview of immunoprecipitation experiment as performed in $\mathbf{g}$ and $\mathbf{h}$ to monitor TOM70-TOM core interaction dependent on TOM70pSer91 phosphorylation (yellow circle). Numerical source data, unprocessed immunoblots and autoradiography scans are reported in the Source Data File.

phosphorylation could regulate association of the peripheral TOM70 receptor with the core TOM complex, which is required for translocation of the precursors across the outer membrane. To test this hypothesis we incubated isolated mitochondria with lambda phosphatase or DYRK1A to ensure non-phosphorylated or largely phosphorylated TOM70, respectively (Supplementary Fig. 5e). Mitochondria were lysed using a mild non-ionic detergent and the entire TOM complex was enriched by coimmunoprecipitation with TOM22 antibodies. TOM22 is a central component of the TOM core complex, which harbors also the translocation channel TOM40 (29). We found an increase of TOM70 binding to the TOM complex when mitochondria had been treated with DYRK1A prior to the purification (Supplementary Fig. 5f). We further validated the phosphorylationdependent stimulation of TOM70 interaction with the TOM core complex in mitochondria purified from mouse brain tissue and also detected a significant increase in TOM70 binding to the TOM complex similarly to mitochondria isolated from cultured cells (Fig. 4g, h).

In summary, DYRK1A phosphorylation at Ser91 does not affect TOM70 biogenesis itself or its interaction with incoming precursor proteins. However, phosphorylation of TOM70 Ser91 strongly stimulates the interaction of the peripheral TOM70 receptor with the TOM core complex (Fig. 4i). This interaction is essential for the transfer of TOM70 receptor-bound precursor proteins to the translocation pore to initiate the import process.

DYRK1A controls the mitochondrial carrier import pathway via TOM70 Ser91. To investigate if TOM70 Ser91 phosphorylation is required to mediate transfer of the precursors to the TOM core complex and thereby initiate translocation across the outer membrane we performed in organello import experiments that allow dissection of the different steps in mitochondrial protein biogenesis. We used TIM23 as model substrate for the TOM70dependent metabolite carrier import pathway and Hsp10 as model precursor to study the presequence import pathway, which does not depend on TOM70 as import receptor ${ }^{24,25,54-56}$ (Fig. $4 \mathrm{~d}$, e). We first assessed both import pathways after removal of the TOM70 Ser91 phosphorylation site by lambda phosphatase treatment (LP, Fig. 5a). The carrier import pathway (monitored by membrane-potential $(\Delta \psi)$ dependent assembly into the inner membrane via Blue Native PAGE ${ }^{24}$ was dramatically reduced upon phosphatase treatment confirming the critical role of TOM70 Ser91 phosphorylation in the carrier import pathway (Fig. 5b, c). In contrast, the presequence import pathway was unaffected (Fig. 5d). 
a

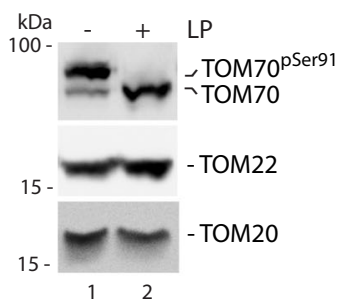

C
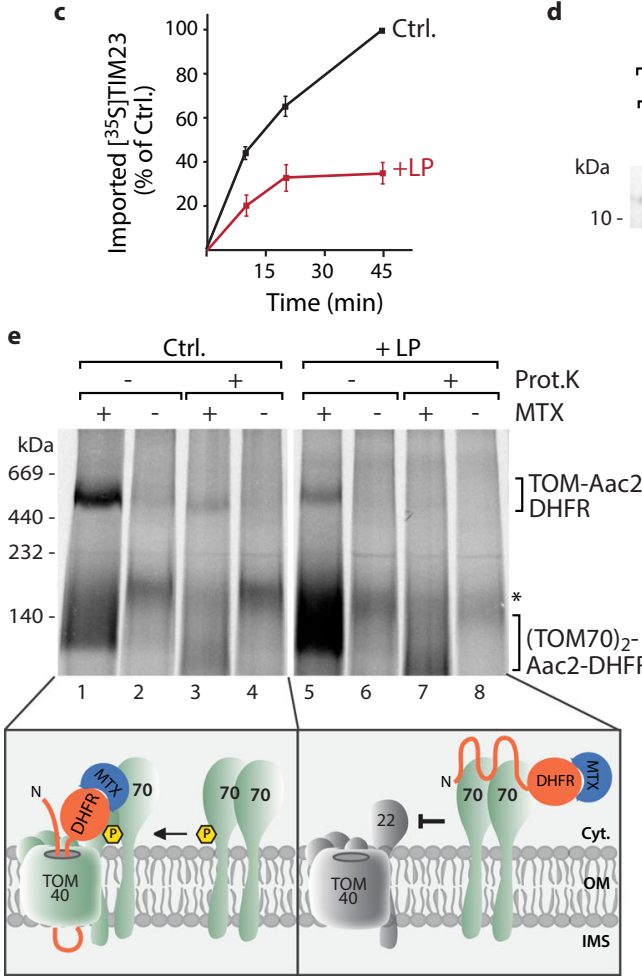

b

d
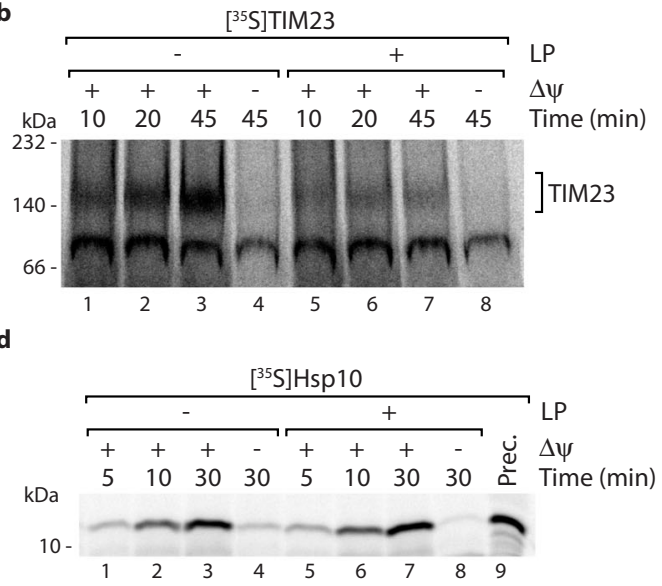

f

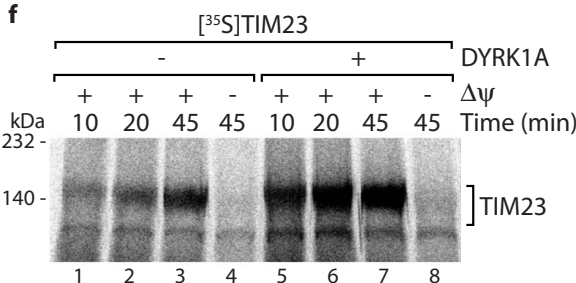

g

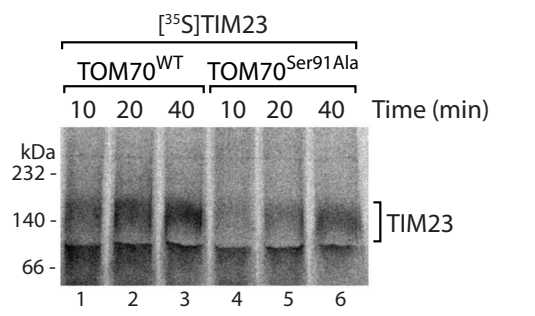

Fig. 5 TOM70Ser91 phosphorylation by DYRK1A activates the metabolite carrier import pathway. a Phos-tag gel analysis of TOM import receptors in mitochondria upon treatment with lambda phosphatase (LP). b Import of radiolabelled TIM23 precursor into isolated mitochondria treated with (+) or without (-) lambda phosphatase (LP) as shown in a. Where indicated the membrane potential $\Delta \psi$ was dissipated prior to the import reaction. Samples were lysed in digitonin buffer and subjected to Blue Native PAGE. Imported protein was analyzed by autoradiography. c Quantification of imported TIM23 from b. Ctrl. at 45 min import time was set to 100\%. Data represent mean \pm SEM from three independent experiments. d Import of radiolabelled Hsp10 precursor (Prec.) into isolated mitochondria that were treated with $(+)$ or without $(-)$ lambda phosphatase (LP). Samples were treated with proteinase K after import and analyzed by SDS-PAGE and autoradiography. e Import of radiolabelled Aac2-DHFR precursor for 25 min into mitochondria that have been treated as in a-d in the presence or absence of Methotrexate (MTX). Samples were separated via Blue Native PAGE and analyzed by autoradiography. Proteinase K (Prot. K) treatment reveals accessibility of arrested precursor at TOM complex or TOM70 dimer from the cytosolic side. *, inner membrane assembled Aac2-DHFR is visible in lanes 2 and 4. Cartoons illustrate requirement of phosphorylation for efficient transfer of precursor from TOM70 to the TOM complex. $\mathbf{f}$ Phosphatase-treated mitochondria were incubated in the presence $(+)$ or absence $(-)$ of DYRK1A followed by import of radiolabelled TIM23 precursor. Reconstitution of TIM23 import was analyzed via Blue Native PAGE and autoradiography. $\mathbf{g}$ TIM23 import analysis in mitochondria from TOM70 knock-down cells reexpressing TOM70WT and TOM70 $591 \mathrm{~A}$ variants. Numerical source data and unprocessed immunoblots and autoradiography scans are reported in the Source Data File.

Import of carrier precursor proteins can be subdivided into different steps: The hydrophobic precursors are bound to cytosolic chaperones, which mediate the initial interaction with the TOM70 receptor. TOM70 binds the precursors and transfers them to the TOM core complex for translocation across the outer membrane. Small Tim chaperones shield the precursor in the intermembrane space and mediate transfer to the TIM22 complex, which inserts the transmembrane helices in the inner membrane in a membrane potential-dependent process $1,3,6,16,17$. To uncover which exact step in the carrier import pathway is affected by removal of TOM70 Ser91 phosphorylation, we imported the ADP/ATP carrier precursor protein fused to a dihydrofolate reductase (DHFR) domain $\left(\left[{ }^{35} \mathrm{~S}\right] \mathrm{Aac} 2-\mathrm{DHFR}\right){ }^{16}$. In the presence of the folate analog methotrexate (MTX) the DHFR domain is tightly folded so that the entire precursor construct arrests at the TOM complex as translocation across the import channel is blocked. Analysis by Blue Native PAGE revealed that in non-treated mitochondria, in which TOM70 Ser91 is phosphorylated, the Aac2-DHFR precursor accumulates at the TOM complex in the presence of MTX (Fig. 5e, lanes 1-4). This illustrates that TOM70 interacts with the TOM core and transfer of the precursor across the TOM pore is initiated. In contrast, phosphatase treatment of mitochondria prior to the import reaction results in arrest of the Aac2-DHFR predominantly at the TOM70 receptor dimer demonstrating that it cannot be transferred to the TOM core complex due to ablation of the TOM70-TOM-interaction by loss of the TOM70 Ser91 phosphorylation (Fig. 5e, lanes 5-8). 
Finally, we wondered if the carrier import pathway can be reactivated by DYRK1A-mediated re-phosphorylation of TOM70 Ser91 (Figs. 1d-f and $4 \mathrm{~h}$, and Supplementary Fig. 1f). Indeed, phosphorylation of this single position at TOM70 by DYRK1A led to a vigorous recovery of TIM23 import in phosphatase-treated mitochondria (Fig. 5f). To directly pin the activity of the TIM23 import pathway to the TOM70 0 Ser91 site we expressed TOM70 $\mathrm{WT}$ or the TOM70 ${ }^{\mathrm{S} 91 \mathrm{~A}}$ variant in cells in which expression of endogenous TOM70 had been reduced by shRNA induction (Fig.3d, e). Analysis of TIM23 assembly revealed that the carrier import pathway was strongly impaired in mitochondria isolated from cells expressing the TOM70 ${ }^{S 91 \mathrm{~A}}$ variant compared to TOM70WT cells (Fig. 5g and Supplementary Fig. 6).

Taken together, DYRK1A-dependent phosphorylation of TOM70 Ser91 emerges as central modification at the human mitochondrial import machinery critically required for activation of the metabolite carrier import pathway.

\section{Discussion}

Our study identifies a regulatory mechanism of the human mitochondrial import machinery by a cytosolic protein kinase. The unexpected interplay between mitochondrial protein biogenesis and the DYRK1A kinase, which has not been linked to mitochondria before, underscores the necessity of unbiased, global approaches to uncover regulatory principles in mitochondrial and cellular biology. Notably, only a very limited fraction of the human kinome is investigated in detail while for hundreds of kinases the biological function remains enigmatic ${ }^{36}$. Our study shows that a systematic approach like the KinaseFinder platform can lead to unforeseen biological links. Impairment of DYRK1A signaling leads to compromised mitochondrial structure and function. DYRK1A exclusively targets the Ser91 position in the peripheral TOM70 receptor and the implications of this phosphorylation on the import process were identified on the molecular level. The modification did not influence biogenesis of the TOM70 receptor itself and unexpectedly did also not impact on its precursor receptor function. In contrast, we found that Ser91 phosphorylation is strongly required to stimulate the transfer of precursors from the receptor-bound state to the translocation pore (Fig. 6). Notably, exactly this step, the transfer of precursors from the TOM70 receptor to the translocation pore, which requires the precursor in a chaperone-bound state, has previously been described as being ATP-dependent. It has been proposed that ATP hydrolysis is required for the release of chaperones from the precursor ${ }^{17,57,58}$. However, our findings offer an exciting alternative explanation, that the ATP dependency is mediated by the protein kinase DYRK1A discovered in this study.
The importance of DYRK1A signaling for functional mitochondria is further underscored by discovery of a protective transcriptional response upon DYRK1A inhibition that includes upregulation of components of the mitochondrial import machinery and of several members of the DYRK family and related kinases, which may act as a safety backup to secure TOM70 Ser91 phosphorylation. It therefore appears that preservation of a functional carrier import pathway via DYRK kinases is an essential process for cell life. Our findings open here an avenue in the converging fields of mitochondrial biogenesis and signal transduction. How the protective transcriptional response discovered in this study is triggered and controlled will be an important research focus in succeeding investigations. Similarly, future studies will be required to link cell-, tissue-, or organ-specific activities of DYRK1A with the necessity to control the mitochondrial import machinery.

Finally, the identification of DYRK1A as a TOM kinase uncovers a connection between DYRK1A signaling and mitochondria. This link opens up the exciting possibility to investigate and decipher the contribution of mitochondrial dysfunction in the many clinical aspects of dysfunctional DYRK1A including DYRK1A-related syndrome, Autism Spectrum Disorder, and Down syndrome ${ }^{37-41}$.

\section{Methods}

Tissue culture and mice. For in vivo and in organello experiments we used human embryonic kidney cells (HEK293T or Flp-In T-REx ${ }^{\mathrm{TM}}$ 293; Thermo Fisher), human bone osteosarcoma epithelial cells (U2OS, Invitrogen), or mouse brain tissue (C57BL6/N). Cells were cultured in DMEM (Gibco) containing 10\% (v/v) fetal bovine serum (FBS; Sigma), $2 \mathrm{mM}$ L-glutamine (Sigma), and $4.5 \mathrm{~g} / \mathrm{l}$ glucose. Cells were grown at $37^{\circ} \mathrm{C}$ in a humidified incubator with $5 \% \mathrm{CO}_{2}$. C57Bl6/ $\mathrm{N}$ mice were bred and maintained in the animal facilities of the University Medical Center Freiburg according to institutional guidelines and in accordance with the German law for animal protection. Mice were kept in specific pathogen-free conditions, fed with standard diet and had access to food and water ad libitum. Temperature in the animal facility was $21-23^{\circ} \mathrm{C}$ with $45-60 \%$ humidity. A day/ night rhythm of $12 \mathrm{~h}$ light and $12 \mathrm{~h}$ darkness was maintained. Tissue sampling from sacrificed mice was approved by the government commission for animal protection and the local ethics committee (University medical center of Freiburg University; X-18/10 C).

Generation of cell lines. For shRNA-mediated depletion of TOM70 the TRIPZ inducible lentiviral non-silencing shRNA system was used (Horizon Discovery, mature antisense: TTATCATACATTTCATCAG, clone ID: V2THS_95541;

RHS4743 for control non-silencing). For generation of TRIPZ shRNA lentivirus, $1.6-2 \times 10^{6}$ HEK293T cells were seeded into $10 \mathrm{~cm}$ tissue culture dishes. On the following day, medium was changed and cells were transfected with Poly-

ethylenimine (PEI, Sigma-Aldrich). PEI transfection was performed in a 1:3 DNAPEI mixture in DMEM without supplements. The mixture was incubated at room temperature for $20 \mathrm{~min}$ and added drop-wise to the cells (1/3 of the total medium volume). Lentivirus containing supernatant was harvested after $48 \mathrm{~h}$, and filtered with a $0.2-\mu \mathrm{m}$ syringe filter. For viral transduction, $5 \times 10^{5} \mathrm{HEK} 293 \mathrm{~T}$ cells were
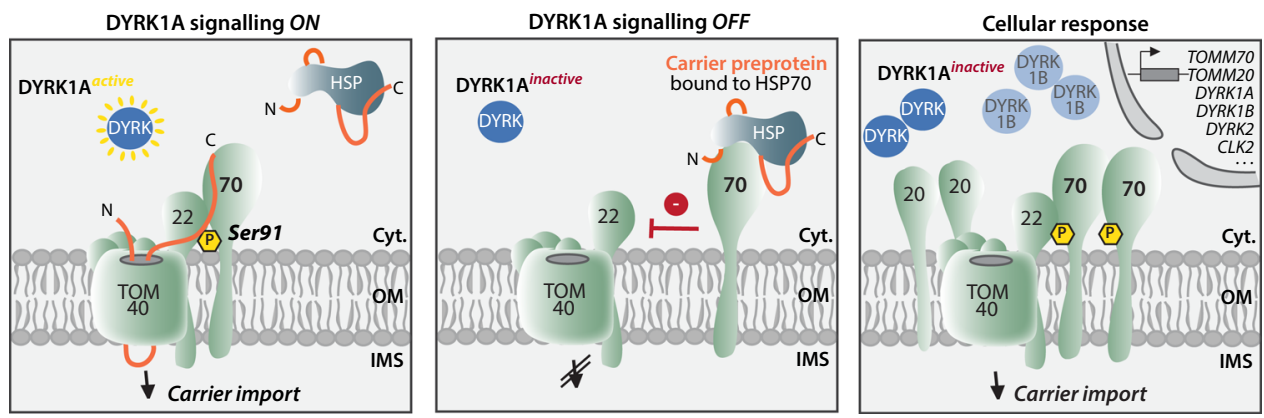

Fig. 6 Schematic model depicting the role of DYRK1A kinase as critical activator of the carrier import pathway in human mitochondria. DYRK1Amediated phosphorylation of TOM70 Ser91 stimulates docking of the precursor bound import receptor to the central translocation pore to enable import of carrier proteins. Upon inhibition, DYRK1A fails to phosphorylate TOM70Ser91 and protein import is impaired. A protective response secures the carrier import pathway by upregulation of TOM70 and further import receptors as well as backup kinases which can also phosphorylate TOM70 ${ }^{\text {Ser } 91}$ to compensate for the lack of DYRK1A. 
seeded in six-well plates. Filtered shRNA lentivirus containing supernatant and fresh DMEM containing $10 \% \mathrm{FBS}$ and $8 \mu \mathrm{g} / \mathrm{ml}$ polybrene (Sigma) were mixed in a 1:1 ratio and added to the cells on the next day. After $24 \mathrm{~h}$, medium was replaced by fresh full DMEM. Afterwards, full DMEM containing $1 \mu \mathrm{g} / \mathrm{ml}$ puromycin (Sigma) was used for selection. One $\mu \mathrm{g} / \mathrm{ml}$ doxycycline hydrochloride (Sigma) was used for induction of TOMM70 gene silencing.

To induce transient overexpression of TOM70 we cloned the cDNA of human TOMM70 WT and Ser91Ala variant into pMIG plasmids. HEK293T cells were then transfected with pMIG-TOM70 or pMIG-TOM70 $0^{\text {S91A }}$ using PEI as described above and harvested after $24 \mathrm{~h}$. For in vivo fluorescence microscopy U2OS cells were transfected with the same vectors using FuGene (Promega) as described by the manufacturer in $27 \mathrm{~mm}$ glass base dishes (Thermo Scientific) and harvested after $48 \mathrm{~h}$.

CRISPR/Cas9 genome editing was used to generate a DYRK1A knockout in the Flp-In T-REx 293 cell line (Thermo Fisher). The required plasmid pSpCas9(BB)2A-GFP (PX458) was a gift from F. Zhang (Addgene plasmid, \#48138) ${ }^{59}$. The guide RNA sequence (GAGAAACACCAATTTCCGAG) targeting exon 6 of human DYRK1A was cloned into pSpCas9 (BB)-2A-GFP vector. Flp-In T-REx 293 cells were transfected using Lipofectamine 2000 (Invitrogen). $5 \mathrm{~h}$ after transfection, GFP-positive cells were single-cell sorted into 96-well plates by FACS. Clones were expanded and screened for DYRK1A expression via immunoblotting (all primary antibodies used in this study are listed in Supplementary Table 1). For the genomic verification of the DYRK1A knockout cell line, the targeted exon 6 was PCRamplified from genomic DNA isolated from individual clones with Quick-gDNA MiniPrep (Zymo Research). PCR products were ligated into PCR'-Blunt expression vector (Thermo Fisher) and indel mutations were verified by sequencing.

Isolation of mitochondria. Mitochondria were isolated as previously described by Johnston et al. ${ }^{60}$. Eight-weeks-old mice were euthanized by cervical dislocation and brain tissue removed. Subsequently, tissues were weighed and cut into small pieces. For isolation of mitochondria from HEK293T cells, cells were harvested. Tissue and cell pellets were resuspended in solution A ( $220 \mathrm{mM}$ mannitol, $70 \mathrm{mM}$ sucrose, $20 \mathrm{mM}$ HEPES-KOH, $\mathrm{pH} 7.6,1 \mathrm{mM}$ EDTA, $0.5 \mathrm{mM}$ PMSF, and $2 \mathrm{mg} / \mathrm{ml} \mathrm{BSA}$ ) and subsequently homogenized using a glass potter. Cellular debris was removed by centrifugation at $800 \times g$ for $5 \mathrm{~min}$ at $4{ }^{\circ} \mathrm{C}$ and mitochondria were pelleted from the supernatant by further centrifugation at $10,000 \times g$ for $15 \mathrm{~min}$ at $4^{\circ} \mathrm{C}$. Crude mitochondrial pellet was resuspended in solution B (solution A without BSA) and again centrifuged at $800 \mathrm{~g}$ for $5 \mathrm{~min}\left(4^{\circ} \mathrm{C}\right)$. Mitochondria were then pelleted by a $10,000 \times g$ spin for $15 \mathrm{~min}$ and resuspended in import buffer (described below). Protein concentrations were determined by Bradford assay and samples stored at $-80^{\circ} \mathrm{C}$ or directly used for functional assays. For in vitro experiments we used soluble cytosolic domains of the human import receptors TOM20 (residues 35-145), TOM22 (residues 2-80), and TOM70 (residues 70-608), which were either generated by expression and affinity purification in E. coli (TOM20 and TOM70, each with $\mathrm{N}$-terminal deca-Histidine tag) or directly synthesized in case of TOM22 (GeneCust).

Expression and purification of soluble TOM receptor domains and Frataxin cDNAs encoding cytosolic domains (cd) of human TOM70, TOM20, and Frataxin (FXN) (full length) were cloned into pET10N vector ${ }^{61}$ providing a deca Histidine tag at the $\mathrm{N}$-terminus of the protein. Point mutations for generating the TOM $70_{c d}{ }^{\mathrm{T} 85 \mathrm{~A}}, \mathrm{TOM} 70_{\mathrm{cd}} \mathrm{S} 91 \mathrm{~A}$, and TOM $70_{\mathrm{cd}}{ }^{\mathrm{S} 110 \mathrm{~A}}$ variants were introduced by sitedirected mutagenesis. Vector constructs were transformed into E. coli and cells were grown at $37^{\circ} \mathrm{C}$ until an $\mathrm{OD}_{600}$ of 0.6. Expression was induced by addition of $1 \mathrm{mM}$ IPTG. After growth for additional $3 \mathrm{~h}, 40 \mathrm{ml}$ of the culture was harvested and pelleted cells were resuspended in extraction buffer $(20 \mathrm{mM}$ Tris/ $\mathrm{HCl}, \mathrm{pH} 8.0$, $4 \mathrm{mM} \mathrm{MgCl} 2,0.5 \mathrm{mM} \mathrm{PMSF}$ ) and incubated with $1 \mathrm{mg} / \mathrm{ml}$ lysozyme and DNase I for $30 \mathrm{~min}$ on ice. Lysate was cleared at $10,000 \times g$ for $10 \mathrm{~min}$ at $4^{\circ} \mathrm{C}$. The supernatant was incubated with Ni-NTA resin beads (Qiagen) and washed three times with washing buffer $(20 \mathrm{mM}$ Tris/HCl, $\mathrm{pH}$ 8.0, $50 \mathrm{mM}$ Imidazole, $200 \mathrm{mM} \mathrm{NaCl}, 1$ $\mathrm{mM} \beta$-mercaptoethanol). Proteins were eluted with elution buffer $(20 \mathrm{mM}$ Tris/ $\mathrm{HCl}, \mathrm{pH}$ 8.0, $350 \mathrm{mM}$ Imidazole, $100 \mathrm{mM} \mathrm{NaCl}, 1 \mathrm{mM} \beta$-mercaptoethanol). Grades of protein purity were analyzed by SDS-PAGE and Coomassie brilliant blue staining.

Phosphorylation assays. For in vitro phosphorylation, purified receptor domains $(2 \mu \mathrm{g})$ were incubated in $30 \mu \mathrm{l} \mathrm{ST}$ buffer $(10 \mathrm{mM}$ Tris/HCl, $\mathrm{pH} 7.2,250 \mathrm{mM}$ sucrose, $2 \mathrm{mM} \mathrm{MgCl}_{2}$, and $1 \mathrm{mM} \mathrm{PMSF}$ ) containing 5-10 mM ATP and $1 \mu \mathrm{l}$ of the respective protein kinase (purchased from Thermo Scientific, NEB or ProQinase). Reactions were incubated for $45 \mathrm{~min}$ at $30^{\circ} \mathrm{C}$ (gentle agitation at $500 \mathrm{rpm}$ ) and subsequently stopped by addition of Laemmli buffer. Samples were analyzed by Phos-tag gels and immunoblotting.

For in organello phosphorylation, isolated mitochondria $(50 \mu \mathrm{g})$ were incubated in $50 \mu \mathrm{l}$ kinase assay buffer $(10 \mathrm{mM}$ Tris/HCl pH 7.4, $250 \mathrm{mM}$ sucrose, $10 \mathrm{mM}$ $\mathrm{MgCl}_{2}, 2 \mathrm{mM}$ PMSF, 1x PhosStop (Roche), 1x EDTA free protease inhibitor cocktail (Roche), and 1x Protein Metallo Phosphatase (PMP) buffer (NEB)). For initial dephosphorylation $1 \mu$ lambda phosphatase (LP, NEB) was added and incubated for $45 \mathrm{~min}$ at $37^{\circ} \mathrm{C}$ (gentle agitation at $550 \mathrm{rpm}$ ). Mitochondria were washed twice with sucrose buffer (10 mM HEPES/HCl, $\mathrm{pH} 7.6,0.5 \mathrm{M}$ sucrose) and resuspended in assay buffer containing 5-10 mM ATP and $1 \mu \mathrm{l}$ of the respective protein kinase (NEB, ProQinase or Thermo Fisher). After incubation for $45 \mathrm{~min}$ at $37^{\circ} \mathrm{C}$ (gentle agitation at $550 \mathrm{rpm}$ ) mitochondria were washed with sucrose buffer and resuspended in Laemmli buffer. Samples were analyzed by Phos-tag gels and immunoblotting.

Kinase inhibition assays. HEK293T or U2OS cells (for in vivo fluorescence microscopy) were cultured in full DMEM media as described above in the presence of $10 \mu \mathrm{M}$ INDY (Tocris Bioscience; $10 \mathrm{mM}$ stock in DMSO) for different periods of time (overnight, $24 \mathrm{~h}, 48 \mathrm{~h}$, or $72 \mathrm{~h}$ ). Cells were subjected to functional assays as described below. For cell growth $5 \times 10^{4}$ cells were seeded and incubated in the presence of $10 \mu \mathrm{M}$ INDY or DMSO as control. Cell numbers were determined using a Neubauer cell counting chamber after $48 \mathrm{~h}$.

To assess inhibition by INDY in vitro, DYRK1A kinase was incubated with different concentrations of INDY $(2-10 \mu \mathrm{M})$ for $30 \mathrm{~min}$ in $30 \mu \mathrm{l}$ ST buffer containing 5-10 mM ATP. Purified TOM $70_{\mathrm{cd}}$ was added and samples further incubated for $45 \mathrm{~min}$ at $30^{\circ} \mathrm{C}$ (gentle agitation at $500 \mathrm{rpm}$ ). Reactions were stopped by addition of Laemmli buffer. Samples were analyzed by Phos-tag gels and immunoblotting.

\section{In vitro transcription/translation and in organello import of precursor pro-} teins. Radiolabelled precursor proteins (human TOM70 $\mathrm{WT}, \mathrm{TOM} 70^{\mathrm{S} 91 \mathrm{~A}}$

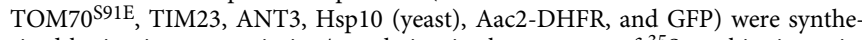
sized by in vitro transcription/translation in the presence of ${ }^{35} \mathrm{~S}$-methionine using the rabbit reticulocyte lysate system (Promega) ${ }^{34,62}$. For import assays, isolated mitochondria and radiolabelled precursor proteins were mixed in import buffer ( $250 \mathrm{mM}$ sucrose, $5 \mathrm{mM}$ magnesium acetate, $80 \mathrm{mM}$ potassium acetate, $10 \mathrm{mM}$ sodium succinate, $20 \mathrm{mM}$ HEPES-KOH, $\mathrm{pH}$ 7.4) supplemented with $1 \mathrm{mM}$ DTT and $5-10 \mathrm{mM}$ ATP and incubated for various time points at $37^{\circ} \mathrm{C}$. For disruption of the membrane potential $\Delta \psi$, antimycin $\mathrm{A}(8 \mu \mathrm{M})$, valinomycin $(1 \mu \mathrm{M})$, and oligomycin $(20 \mu \mathrm{M})$ were added prior to the import reaction. To remove endogenous phosphorylation sites, isolated mitochondria were incubated with LP for 30 min in ST buffer before the import reaction. For folding of the DHFR domain to block Aac2-DHFR precursor translocation methotrexate $(20 \mu \mathrm{M})$ was added prior to the import reaction ${ }^{16}$. For removal of non-imported precursor proteins samples were treated with $20 \mu \mathrm{g} / \mathrm{ml}$ Proteinase K. Mitochondria were re-isolated by centrifugation at $10,000 \times g$ for $10 \mathrm{~min}$ at $4{ }^{\circ} \mathrm{C}$ and samples were analyzed by SDSPAGE and autoradiography ${ }^{62}$. For analyzing protein assembly, mitochondrial pellets were lysed in digitonin buffer $(0.4-1 \%(\mathrm{w} / \mathrm{v})$ digitonin, $0.5 \mathrm{mM}$ EDTA, $10 \%$ (w/v) glycerol, $50 \mathrm{mM} \mathrm{NaCl}, 20 \mathrm{mM}$ Tris- $\mathrm{HCl}, \mathrm{pH}$ 7.4) and incubated on ice for 15 min. After clarifying spin at $20,000 \times g$ for $5 \mathrm{~min}$ at $4{ }^{\circ} \mathrm{C}$, supernatant was loaded on gradient Blue-native PAGE $(4-13 \%$ or $6-16 \%(\mathrm{w} / \mathrm{v})$ acrylamide) followed by autoradiography or immunoblotting ${ }^{16,34,60}$. Images were processed using ImageJ (1.52) software.

Phos-tag electrophoresis. $\mathrm{Zn}^{2+}$-Phos-tag Bis-Tris gel electrophoresis was performed as described previously ${ }^{48}$. Briefly, discontinuous $8 \%$ or $12.5 \%$ SDS-PAGE was prepared with the modification that $12.5 \mu \mathrm{M}$ Phos-tag reagent and $25 \mu \mathrm{M}$ $\mathrm{ZnCl}_{2}$ were added to the separation gel mix before polymerization ( $50 \mu \mathrm{M}$ Phos-tag and $100 \mu \mathrm{M} \mathrm{ZnCl}_{2}$ for analysis of TOM20 and TOM22 phosphorylation). Electrophoresis was performed for $3-4 \mathrm{~h}$ at $35 \mathrm{~mA}$ and $600 \mathrm{~V}$. Afterwards, wet transfer process (Biorad system) was used for protein transfer onto a PVDF membrane. Proteins were detected via immunodecoration.

Precursor binding assay. Purified receptor domains with $\mathrm{N}$-terminal deca-His tags or control proteins that had been incubated in the presence or absence of DYRK1A as described above were resuspended with $\mathrm{Ni}^{2}+$-NTA resin equilibrated with assay buffer $(20 \mathrm{mM}$ Imidazole, $10 \mathrm{mM} \mathrm{KCl}, 10 \mathrm{mM} \mathrm{MOPS} / \mathrm{KOH}, \mathrm{pH} 7.2,1 \%$ (w/v) BSA). After incubation for $30 \mathrm{~min}\left(4^{\circ} \mathrm{C}\right)$ resin was transferred to $1 \mathrm{ml}$ Mobicol mini column. $\left.{ }^{35} \mathrm{~S}\right]$-labeled precursor or control proteins were diluted in assay buffer (up to max. $5 \%(\mathrm{v} / \mathrm{v})$ rabbit reticulocyte lysate was used) and added to the resuspended resin. After incubation for $30 \mathrm{~min}$ at $30^{\circ} \mathrm{C}$ resin was pelleted and washed three times with assay buffer without BSA. Bound proteins were eluted with Laemmli buffer and analyzed by SDS-PAGE, autoradiography, and immunoblotting.

Co-immunoprecipitation of the TOM complex. Mitochondria were isolated from mouse brain tissue or HEK293T cells and incubated either with DYRK1A or Lambda phosphatase as described above to achieve a maximally phosphorylated or non-phosphorylated state of TOM70. Samples were then lysed in $0.5 \%(\mathrm{w} / \mathrm{v})$ Digitonin buffer with $1 \mathrm{x}$ PhosStop (Roche) and 1x EDTA free protease inhibitor cocktail (Roche). Lysates were cleared by centrifugation at $20,800 \times g$ for $10 \mathrm{~min}$ at $4^{\circ} \mathrm{C}$. Supernatants were incubated with pre-coupled anti-TOM 22 serum to Protein A-Sepharose beads (GE Healthcare). After incubation for $40 \mathrm{~min}$ at $4^{\circ} \mathrm{C}$, the resin was collected by centrifugation and washed six times with $0.1 \%$ digitonin buffer. Bound proteins were eluted with Laemmli buffer and subjected to SDS-PAGE or Phos-tag gels and immunoblot analysis. 
Measurement of respiratory activity. Mitochondrial respiratory activity was measured using a XF96 Extracellular Flux analyzer (Seahorse Bioscience). Cells were seeded at a density of $2 \times 10^{4}$ cells/well on a poly-D-lysine coated plate one day before the measurement. Basal levels of oxygen consumption rate (OCR) and OCR in the presence of electron transport chain inhibitors and uncouplers $(2 \mu \mathrm{M}$ oligomycin, $0.3 \mu \mathrm{M}$ carbonyl cyanide-4-(trifluoromethoxy) phenylhydrazone, $1 \mu \mathrm{M}$ antimycin $\mathrm{A}$ and $1 \mu \mathrm{M}$ rotenone) were measured. OCR values were normalized to the protein content (determined after the measurement by Bradford assay).

In vivo fluorescence microscopy. U2OS cells were grown in $27 \mathrm{~mm}$ glass base dishes (see above) and incubated in the absence or presence of INDY $(10 \mu \mathrm{M})$ for $24 \mathrm{~h}$. Prior to visualization, cells were stained for $30 \mathrm{~min}$ with $50 \mathrm{nM}$ MitoTracker Red CMXRos (Thermo Scientific) in culture media. Fluorescent microscopy images were recorded with a DeltaVision Ultra High Resolution Microscope with UPlanSApo 100x/1.4 oil Olympus objective, using a sCMOS pro.edge camera at $37^{\circ} \mathrm{C}$. Raw fluorescent microscopy images were deconvolved at the DeltaVision microscope using SoftWorx deconvolution plugin. Quantification of morphological parameters of mitochondria was performed with the ImageJ 1.52 software (Wayne Rasband, National Institutes of Health, USA), using the Analyze Particles functionality ${ }^{63}$. Elongation was calculated as the inverse of circularity. At least 50 cells were analyzed for each condition.

\section{In vitro $\gamma$-[ $\left[{ }^{18} \mathrm{O}_{4}\right]$-ATP kinase assay and MS analysis. Isolated mitochondria} were dephosphorylated by LP treatment as described above. For rephosphorylation $40 \mathrm{ng}$ DYRK1A/ $\mu \mathrm{g}$ mitochondria was added and incubated in kinase assay buffer in the presence of $6 \mathrm{mM} \gamma$ - $\left[{ }^{18} \mathrm{O}_{4}\right]$-ATP (Cambridge Isotope Laboratory), $0.5 \mathrm{mM}$ sodium orthovanadate, and $2.5 \mathrm{mM}$ sodium fluoride for 60 min at $37^{\circ} \mathrm{C}$ with gentle agitation at $550 \mathrm{rpm}$. Samples were washed with sucrose buffer and centrifuged at $10,000 \times g$ for $10 \mathrm{~min}$ at $4^{\circ} \mathrm{C}$. Pellets were snap-frozen and stored at $-80^{\circ} \mathrm{C}$. Samples were lysed in $8 \mathrm{M}$ urea and transferred to $10 \mathrm{kDa}$ MW-cutoff filters (Sartorius). Trypsin digestion was performed overnight according to Filter Aided Sample Preparation (FASP) ${ }^{64}$. On the next day, the peptides were eluted twice with $100 \mu \mathrm{L}$ of $50 \mathrm{mM}$ ammonium bicarbonate. The eluates were acidified with $6 \mu \mathrm{l}$ of $50 \%$ TFA and lyophilized. Dry peptides were resuspended in $200 \mu \mathrm{l} 80 \%$ acetonitrile/1\% TFA and incubated with $2 \mathrm{mg}$ of $\mathrm{TiO}_{2}$ (GL Sciences) for $30 \mathrm{~min}$, which were equilibrated with $300 \mathrm{mg} / \mathrm{ml} \mathrm{lactic}$ acid in $80 \%$ acetonitrile $/ 1 \% \mathrm{TFA}^{65}$. $\mathrm{TiO}_{2}$ beads were washed with $200 \mu \mathrm{l}$ of $10 \%$ acetonitrile/1\% TFA, $200 \mu \mathrm{l}$ of $80 \%$ acetonitrile/1\% TFA, and $50 \mu \mathrm{LC}$-MS grade water. Phosphopeptides were eluted with $50 \mu \mathrm{l}$ of $1 \%$ ammonia in $20 \%$ acetonitrile and $50 \mu \mathrm{l}$ of $1 \%$ ammonia in $40 \%$ acetonitrile. Flow-throughs were measured for nonphosphopeptide analyses.

LC-MS/MS measurements were performed on a QE HF-X mass spectrometer coupled to an EasyLC 1200 nanoflow-HPLC (all Thermo Scientific) essentially as described before ${ }^{64}$. The mass spectrometer was operated in the data-dependent mode; after each MS scan (mass range $m / z=370-1750$; resolution: 120,000) a maximum of twelve MS/MS scans were performed using a normalized collision energy of $25 \%$, a target value of 5,000 and a resolution of 30,000. MS raw files were analyzed using MaxQuant (version 1.6.2.10) ${ }^{66}$ using a Uniprot full-length Homo sapiens (Human) database (March 2016) and common contaminants such as keratins and enzymes used for in-gel digestion as reference.

Carbamidomethylcysteine was set as fixed modification and protein aminoterminal acetylation, serine-, threonine-, and tyrosine- heavy phosphorylation, and oxidation of methionine were set as variable modifications. The MS/MS tolerance was set to $20 \mathrm{ppm}$ and three missed cleavages were allowed using trypsin/P as enzyme specificity. Peptide, site, and protein false discovery rate based on a forward-reverse database were set to 0.01 , minimum peptide length was set to 7 , the minimum score for modified peptides was 40 , and minimum number of peptides for identification of proteins was set to one, which must be unique.

Radiometric filter binding assay. In vitro kinase screening was performed for 339 human protein kinases ( $245 \mathrm{Ser} / \mathrm{Thr}$ kinases and $94 \mathrm{Tyr}$ kinases) using 96-well plates and $5 \mu \mathrm{g}$ purified human $\mathrm{TOM} 70_{\mathrm{cd}}$ or $\mathrm{TOM} 70_{\mathrm{cd}} \mathrm{S} 91 \mathrm{~A}$ protein per well in the presence of $1 \mu \mathrm{M} \gamma$-[ $\left.{ }^{33} \mathrm{P}\right]$-ATP $\left(9.08 \times 10^{05} \mathrm{cpm}\right.$ per well $)$ according to the manufacturer's protocol $^{46}$. Screening was performed by ProQinase (Freiburg, Germany). Values for each kinase were given as counting of cpms of incorporated ${ }^{33}{ }_{\mathrm{Pi}}$ and autophosphorylation activity (in the absence of TOM7 $0_{c d}$ or TOM $70_{c d} \mathrm{~S} 91 \mathrm{~A}$ ) was subtracted. A list of the tested kinases is available at https:/www.proqinase.com/sites/default/files/ public/uploads/KinaseFinder/pq_list_kinasefinder_339_sty_protein_kinases_v03.pdf. The two top score kinases for TOM70 $0_{\text {cd }}$, DYRK1A and DYRK1B, both with activity ratios $>70$ were further validated by titrating the substrate from $0,1.25,2.5$ and $5 \mu \mathrm{g}$ to reveal a substrate concentration-dependent kinase activity.

Identification of TOM70 phosphorylation sites in HEK293T cells. Isolated mitochondria were lysed in three consecutive cycles of snap-freezing $\left(\mathrm{N}_{2}\right.$ liquid), thawing and $1 \mathrm{~min}$ incubation in an ultrasonic bath in presence of $4 \%$ SDS in $50 \mathrm{mM}$ Tris/ $\mathrm{HCl}$ and $150 \mathrm{mM} \mathrm{NaCl}, \mathrm{pH} 7.8$ including $1 \mathrm{x}$ PhosStop (Roche) and $1 \mathrm{x}$ EDTA free protease inhibitor cocktail (Roche). Proteins were reduced by adding DTT to a final concentration of $10 \mathrm{mM}$ and incubation for $30 \mathrm{~min}$ at $56^{\circ} \mathrm{C}$ followed by alkylation via iodoacetamide using a final concentration of $20 \mathrm{mM}$ for $30 \mathrm{~min}$ in the dark

Proteins were precipitated by adding 9 volumes of ice-cold ethanol and incubation at $-40^{\circ} \mathrm{C}$ for $1 \mathrm{~h}$. Precipitated proteins were spun down at $12,000 \times g$ for $40 \mathrm{~min}$ at $4{ }^{\circ} \mathrm{C}$ and pellets were washed using $100 \mu \mathrm{l}$ of ice-cold acetone. Protein pellets were solubilized in $6 \mathrm{M}$ guanidinium- $\mathrm{HCl}$ and the protein concentration was determined using a bicinchoninic acid assay (Pierce, Thermo Scientific). Prior proteolytic digestion, samples were diluted 20-fold using $50 \mathrm{mM}$ ammonium bicarbonate, $1.5 \mathrm{mM} \mathrm{CaCl}_{2}$. Trypsin (Sequencing grade, Promega,) was added in a ratio of 1:20 (trypsin:protein, w/w) and samples were incubated for $13 \mathrm{~h}$ at $37^{\circ} \mathrm{C}$ under slight agitation. The digestion was stopped by addition of FA to a final concentration of $1 \%$ and peptides were purified using SPEC-C18 AR cartridges (Agilent). Digestion quality was controlled via a monolithic column-HPLC, as described previously ${ }^{67}$.

Electrostatic repulsion-hydrophilic interaction liquid chromatography (ERLIC) was performed similarly to Loroch et al. ${ }^{68}$ using an Ultimate 3000 HPLC system (Thermo Scientific) equipped with a PolyWAX (weak anion exchange) column (4.6 mm $\times ; 100 \mathrm{~mm}, 5 \mu \mathrm{m}, 300 \AA$, Poly LC, Columbia) eluted with a binary gradient of $20 \mathrm{mM}$ sodium methylphosphonic acid, $70 \%$ acetonitrile, pH 2 (ERLIC buffer A) and $200 \mathrm{mM}$ triethylammonium phosphate, $60 \% \mathrm{ACN}, \mathrm{pH} 2$ (ERLIC buffer B). Separation was conducted at a flow rate of $1 \mathrm{~mL} / \mathrm{min}$ starting with $100 \%$ A for $10 \mathrm{~min}$ followed by a linear increase to $100 \%$ B over $10 \mathrm{~min}$ followed by $100 \%$ B for 5 min. 24 fractions of $1 \mathrm{~min}$ were collected. To remove acetonitrile and residual ERLIC buffer salts, fractions were dried, reconstituted in $0.1 \%$ TFA, subjected to RP-SPE using Hypersep SpinTips (Thermo Scientific) followed by LC-MS.

LC-MS was done using an LTQ Orbitrap Velos Pro mass spectrometer online coupled to a U3000 RSLC nanoHPLC equipped with an Acclaim PepMap trapcolumn $(100 \mu \mathrm{m} \times ; 2 \mathrm{~cm}, 5 \mu \mathrm{m}$ particles, $100 \AA$ pores $)$ and an Acclaim PepMap main column $(75 \mu \mathrm{m} \times ; 15 \mathrm{~cm}, 2 \mu \mathrm{m}$ particles, $100 \AA$ pores $)$. After $5 \mathrm{~min}$ the trap column was switched in-line and peptides were separated on the main column at a flow rate of $250 \mathrm{nl} / \mathrm{min}$ using a linear acetonitrile gradient $(2.5-30 \%)$ in presence of $0.1 \%$ FA. Gradient lengths were adjusted to the complexity of the fractions: $160 \mathrm{~min}$ for fraction $1-3 ; 100 \mathrm{~min}$ for fraction $4 ; 50 \mathrm{~min}$ for fractions $5-15$; and 20 min for fraction 16-24. The column effluent was introduced to the MS using an NSI source equipped with a $10-\mu \mathrm{m}$ PicoTip emitter (New objective) using a voltage of $1.5 \mathrm{kV}$. The MS was operated in data-dependant acquisition mode with a survey scan acquired in the orbitrap at a resolution $\mathrm{R}=60,000$ followed by up to $15 \mathrm{MS} /$ MS of the most intense precursor ions with a charge of +2 to +4 in the linear ion trap using collision-induced dissociation (top15 CID). The maximum ion injection time was $100 \mathrm{~ms}$ and the AGC target values were set to $10^{6}$ and $10^{4}$ for MS and $\mathrm{MS} / \mathrm{MS}$, respectively. Ions were excluded from re-fragmentation for $20 \mathrm{~s}$ (dynamic exclusion).

For data analysis, raw-files were analyzed using Proteome Discoverer v1.4. Database search was conducted using Mascot v2.4 against Uniprot, taxonomy human (www.uniprot.org, 11-2016, 20,120 target sequences). DB search parameters were: trypsin with two missed cleavage sites; carbamidomethylation of cysteine as fixed modification; oxidation of methionine, $\mathrm{N}$-terminal acetylation and phosphorylation of serine, threonine, and tyrosine as variable modification. Error tolerances were set to $10 \mathrm{ppm}$ and $0.5 \mathrm{Da}$ for precursor and fragment ions, respectively. phosphoRS v3.1 was used to assign phosphosite localization probabilities $^{69}$ and results were filtered to meet a $1 \%$ false discovery rate (on PSM level) using the Percolator 2.04 node $^{70}$. Phosphosites were considered identified if the phosphoRS site probability was $\geq 90 \%$. We identified 212 phosphorylation sites derived from 101 mitochondrial proteins (filtered against MitoCarta 2.0) of which three phosphopeptides derived from TOM70 (Supplementary Fig. S1C). The mass spectrometry proteomics data have been deposited to the ProteomeXchange Consortium via the $\mathrm{PRIDE}^{71}$ partner repository with the dataset identifier PXD019520.

RNA isolation and qRT-PCR. Total RNA was isolated using the RNeasy Mini Kit (Qiagen) with DNase treatment to omit DNA contaminations. cDNA was synthesized with High-Capacity cDNA kit (Applied Biosystems) from $2 \mu \mathrm{g}$ RNA. PCR amplifications and detections were performed with CFX Connect Real-time PCR detection system (Bio-Rad) using SsoAdvanced Universal SYBR Green Supermix (Bio-Rad). $\beta$-Actin (ACTB) was used as housekeeping gene for normalization. Relative mRNA levels were calculated with the delta-delta Ct method. A complete list of primers is provided in Supplementary Table 2.

Statistics and reproducibility. Data shown represent means \pm standard error of the mean (SEM). Statistical details of each experiment can be found in the figure legends. A two-sided Student's $t$ test was applied to compare between two groups A one-way ANOVA followed by a Bonferroni post-hoc test was used to allow multiple comparisons. The level of significance is indicated in the figures with asterisks $(* * * p<0.001, * * p<0.01, * p<0.05$, not significant (n.s.) $p>0.05$ ) and is provided in the figure legends as exact $\mathrm{p}$-value as obtained by the indicated statistic test. Multiple independent experiments were carried out for each experiment and all biochemical experiments were replicated at least three times and obtained the same results. Respiratory activity was determined in two biological replicates (six technical replicates each) and obtained the same results. Gene expression analysis (three replicates in each measurement) and in vivo microscopy analyses were 
performed from two biological replicates in independent experiments and were reproducible.

Reporting summary. Further information on research design is available in the Nature Research Reporting Summary linked to this article.

\section{Data availability}

MS/MS data are deposited to the ProteomeXchange Consortium via the PRIDE partner repository (https://www.ebi.ac.uk/pride) with the dataset identifier PXD019520. Source data and uncropped versions of gels, autoradiography scans and immunoblots are provided in the Source Data File. Further data and resources from this study are available from the corresponding author upon reasonable request.

Received: 7 August 2020; Accepted: 15 June 2021;

Published online: 13 July 2021

\section{References}

1. Neupert, W. \& Herrmann, J. M. Translocation of proteins into mitochondria. Annu. Rev. Biochem. 76, 723-749 (2007).

2. Pagliarini, D. J. et al. A mitochondrial protein compendium elucidates complex I disease biology. Cell 134, 112-123 (2008).

3. Chacinska, A., Koehler, C. M., Milenkovic, D., Lithgow, T. \& Pfanner, N. Importing mitochondrial proteins: machineries and mechanisms. Cell 138, 628-644 (2009).

4. Meisinger, C., Sickmann, A. \& Pfanner, N. The mitochondrial proteome: from inventory to function. Cell 134, 22-24 (2008).

5. Calvo, S. E. \& Mootha, V. K. The mitochondrial proteome and human disease. Annu. Rev. Genomics Hum. Genet. 11, 25-44 (2010).

6. Harbauer, A. B., Zahedi, R. P., Sickmann, A., Pfanner, N. \& Meisinger, C. The protein import machinery of mitochondria - a regulatory hub in metabolism, stress, and disease. Cell Metab. 19, 357-372 (2014).

7. Endo, T. \& Yamano, K. Multiple pathways for mitochondrial protein traffic. Biol. Chem. 390, 723-730 (2009).

8. Vögtle, F. N. et al. Global analysis of the mitochondrial N-Proteome identifies a processing peptidase critical for protein stability. Cell 139, 428-439 (2009).

9. Araiso, Y. et al. Structure of the mitochondrial import gate reveals distinct preprotein paths. Nature 575, 395-401 (2019).

10. Habich, M., Salscheider, S. L. \& Riemer, J. Cysteine residues in mitochondrial intermembrane space proteins: more than just import. Br. J. Pharmacol. 176, 514-531 (2019).

11. Tucker, K. \& Park, E. Cryo-EM structure of the mitochondrial protein-import channel TOM complex at near-atomic resolution. Nat. Struct. Mol. Biol. 26, 1158-1166 (2019).

12. Drwesh, L. \& Rapaport, D. Biogenesis pathways of a-helical mitochondrial outer membrane proteins. Biol. Chem. 401, 677-686 (2020).

13. Schneider, A. Evolution of mitochondrial protein import - lessons from trypanosomes. Biol. Chem. 401, 663-676 (2020).

14. Vögtle, F. N. et al. Landscape of submitochondrial protein distribution. Nat. Commun. 8, 290 (2017).

15. Brix, J., Dietmeier, K. \& Pfanner, N. Differential recognition of preproteins by the purified cytosolic domains of the mitochondrial import receptors Tom 20, Tom22, and Tom70. J. Biol. Chem. 272, 20730-20735 (1997)

16. Wiedemann, N., Pfanner, N. \& Ryan, M. T. The three modules of ADP/ATP carrier cooperate in receptor recruitment and translocation into mitochondria. EMBO J. 20, 951-960 (2001)

17. Young, J. C., Hoogenraad, N. J. \& Hartl, F. U. Molecular chaperones Hsp90 and Hsp70 deliver preproteins to the mitochondrial import receptor Tom70. Cell 112, 41-50 (2003).

18. Palmieri, F. Diseases caused by defects of mitochondrial carriers: A review. Biochim. Biophys. Acta 1777, 564-578 (2008).

19. Yamamoto, H. et al. Roles of Tom70 in import of presequence-containing mitochondrial proteins. J. Biol. Chem. 284, 31635-31646 (2009).

20. MacPherson, L. \& Tokatlidis, K. Protein trafficking in the mitochondrial intermembrane space: mechanisms and links to human disease. Biochem. J. 474, 2533-2545 (2018)

21. Backes, S. et al. Tom70 enhances mitochondrial preprotein import efficiency by binding to internal targeting sequences. J. Cell Biol. 217, 1369-1382 (2018).

22. Sirrenberg, C., Bauer, M. F., Guiard, B., Neupert, W. \& Brunner, M. Import of carrier proteins into the mitochondrial inner membrane mediated by Tim 22 . Nature 384, 582-585 (1996).

23. Rehling, P. et al. Protein insertion into the mitochondrial inner membrane by a twin-pore translocase. Science 299, 1747-1751 (2006)

24. Kang, Y. et al. Sengers Syndrome-associated mitochondrial acylglycerol kinase is a subunit of the human TIM22 protein import complex. Mol. Cell 67, 457-470 (2017).
25. Vukotic, M. et al. Acylglycerol kinase mutated in Sengers Syndrome is a subunit of the TIM22 protein translocase in mitochondria. Mol. Cell 67, 471-483 (2017)

26. Schulz, C., Schendzielorz, A. \& Rehling, P. Unlocking the presequence import pathway. Trends Cell Biol. 25, 265-175 (2015).

27. Hansen, K. G. \& Herrmann, J. M. Transport of proteins into mitochondria. Protein J. 38, 330-342 (2019).

28. Callegari, S., Cruz-Zaragoza, L. D. \& Rehling, P. From TOM to the TIM23 complex - handing over a precursor. Biol. Chem. 401, 709-721 (2020).

29. Meisinger, C. et al. Protein import channel of the outer mitochondrial membrane: a highly stable Tom 40 -Tom 22 core structure differentially interacts with preproteins, small Tom proteins, and import receptors. Mol. Cell. Biol. 21, 2337-2348 (2001).

30. Chan, N. C., Likic, V. A., Waller, R. F., Mulhern, T. D. \& Lithgow, T. The C terminal TPR domain of Tom70 defines a family of mitochondrial protein import receptors found only in animals and fungi. J. Mol. Biol. 358, 1010-1022 (2006)

31. Fan, A. C. Y. et al. Interaction between the human mitochondrial import receptors Tom 20 and Tom70 in vitro suggests a chaperone displacement mechanism. J. Biol. Chem. 286, 32208-32219 (2011).

32. Melin, J. et al. A presequence-binding groove in Tom70 supports import of Mdl1 into mitochondria. Biochim. Biophys. Acta 1853, 1850-1859 (2015).

33. Schmidt, O. et al. Regulation of mitochondrial protein import by cytosolic kinases. Cell 144, 227-239 (2011).

34. Gerbeth, C. et al. Glucose-induced regulation of protein import receptor Tom 22 by cytosolic and mitochondria-bound kinases. Cell Metab. 18, 578-587 (2013).

35. Harbauer, A. B. et al. Mitochondria. Cell cycle-dependent regulation of mitochondrial preprotein translocase. Science 346, 1109-1113 (2014).

36. Edwards, A. M. et al. Too many roads not taken. Nature 470, 163-165 (2011).

37. Park, J., Song, W. J. \& Chung, K. C. Function and regulation of Dyrk1A: towards understanding Down syndrome. Cell. Mol. Life Sci. 66, 3235-3240 (2009).

38. Yamamoto, T. et al. Clinical manifestations of the deletion of Down syndrome critical region including DYRK1A and KCNJ6. Am. J. Med. Genet. A 155A, 113-119 (2011).

39. Wegiel, J., Gong, C. X. \& Hwang, Y. W. The role of DYRK1A in neurodegenerative diseases. FEBS J. 278, 236245 (2011)

40. van Bon, B. W. et al. Intragenic deletion in DYRK1A leads to mental retardation and primary microcephaly. Clin. Genet. 79, 296-299 (2011).

41. Courcet, J. B. et al. The DYRK1A gene is a cause of syndromic intellectual disability with severe microcephaly and epilepsy. J. Med. Genet. 49, 731-736 (2012).

42. Ji, J. et al. DYRK1A haploinsufficiency causes a new recognizable syndrome with microcephaly, intellectual disability, speech impairment, and distinct facies. Eur. J. Hum. Genet. 23, 1473-1481 (2015).

43. Zeviani, M. The expanding spectrum of nuclear gene mutations in mitochondrial disorders. Semin. Cell Dev. Biol. 12, 407-416 (2001).

44. Nunnari, J. \& Suomalainen, A. Mitochondria: in sickness and in health. Cell 148, 1145-1159 (2012).

45. Pagliarini, D. J. \& Rutter, J. Hallmarks of a new era in mitochondrial biochemistry. Genes Dev. 27, 2615-2627 (2013).

46. Butkevich, E. et al. Phosphorylation of FEZ1 by microtubule affinity regulating kinases regulates its function in presynaptic protein trafficking. Sci. Rep. 6, 26965 (2016)

47. Kinoshita, E., Kinoshita-Kikuta, E., Takiyama, K. \& Koike, T. Phosphatebinding tag, a new tool to visualize phosphorylated proteins. Mol. Cell Proteom. 5, 749-757 (2006).

48. Walter, C. et al. Advanced tools for the analysis of protein phosphorylation in yeast mitochondria. Anal. Biochem. 554, 23-27 (2018).

49. Soppa, U. \& Becker, W. DYRK protein kinases. Curr. Biol. 25, R483-R489 (2015).

50. Fernández-Martinez, P., Zahonero, C. \& Sánchez-Gómez, P. DYRK1A: the doubleedged kinase as a protagonist in cell growth and tumorigenesis. Mol. Cell. Oncol. 2, e970048 (2015).

51. Schmitz, M. L., Rodriguez-Gil, A. \& Hornung, J. Integration of stress signals by homeodomain interacting protein kinases. Biol. Chem. 295, 375-386 (2014).

52. Willsey, H. R. et al. Neurodevelopmental disorder risk gene DYRK1A is required for ciliogenesis and brain size in Xenopus embryos. Development https://doi.org/10.1242/dev.189290 (2020).

53. Ogawa, Y. et al. Development of a novel selective inhibitor of the Down syndrome-related kinase Dyrk1A. Nat. Commun. 1, 86 (2010).

54. Gomkale, R. et al. Defining the substrate spectrum of the TIM22 complex identifies pyruvate carrier subunits as unconventional cargos. Curr. Biol. 30, 1119-1127 (2020).

55. Mossmann, D. et al. Amyloid- $\beta$ peptide induces mitochondrial dysfunction by inhibition of preprotein maturation. Cell Metab. 20, 662-669 (2014). 
56. Kücükköse, C. et al. Functional coupling of presequence processing and degradation in human mitochondria. FEBS J. https://doi.org/10.1111/ febs.15358 (2020).

57. Hachiya, N. et al. Reconstitution of the initial steps of mitochondrial protein import. Nature 376, 705-709 (1995).

58. Ellis, R. J. Plugging the transport gap. Nature 421, 801-802 (2003).

59. Ran, F. A. et al. Genome engineering using the CRISPR-Cas9 system. Nat. Protoc. 8, 2281-2308 (2013).

60. Johnston, A. J. et al. Insertion and assembly of human tom7 into the preprotein translocase complex of the outer mitochondrial membrane. J. Biol. Chem. 277, 42197-42204 (2002).

61. Truscott, K. N. et al. A presequence- and voltage-sensitive channel of the mitochondrial preprotein translocase formed by Tim23. Nat. Struct. Biol. 8 1074-1082 (2001).

62. Stojanovski, D., Pfanner, N. \& Wiedemann, N. Import of proteins into mitochondria. Methods Cell Biol. 80, 783-806 (2007).

63. Wiemerslage, L. \& Lee, D. Quantification of mitochondrial morphology in neurites of dopaminergic neurons using multiple parameters. J. Neurosci. Methods 262, 56-65 (2016).

64. $\mathrm{Hu}, \mathrm{Z}$. et al. Multilayered control of protein turnover by TORC1 and Atg1. Cell Rep. 28, 3486-3496 (2019).

65. Zarei, M., Sprenger, A., Rackiewicz, M. \& Dengjel, J. Fast and easy phosphopeptide fractionation by combinatorial ERLIC-SCX solid-phase extraction for in-depth phosphoproteome analysis. Nat. Protoc. 11, 37-45 (2016).

66. Cox, J. \& Mann, M. MaxQuant enables high peptide identification rates, individualized p.p.b.-range mass accuracies and proteome-wide protein quantification. Nat. Biotechnol. 26, 1367-1372 (2008).

67. Burkhart, J. M., Schumbrutzki, C., Wortelkamp, S., Sickmann, A. \& Zahedi, R. P. Systematic and quantitative comparison of digest efficiency and specificity reveals the impact of trypsin quality on MS-based proteomics. J. Proteom. 75, 1454-1462 (2012).

68. Loroch, S., Schommartz, T., Brune, W., Zahedi, R. P. \& Sickmann, A. Multidimensional electrostatic repulsion-hydrophilic interaction chromatography (ERLIC) for quantitative analysis of the proteome and phosphoproteome in clinical and biomedical research. Biochim. Biophys. Acta 1854, 460-468 (2015).

69. Taus, T. et al. Universal and confident phosphorylation site localization using phosphoRS. J. Proteome Res. 10, 5354-5362 (2011).

70. Kall, L., Canterbury, J. D., Weston, J., Noble, W. S. \& MacCoss, M. J. Semisupervised learning for peptide identification from shotgun proteomics datasets. Nat. Methods 4, 923-925 (2007).

71. Perez-Riverol, Y. et al. The PRIDE database and related tools and resources in 2019: improving support for quantification data. Nucleic Acids Res 47, D442-D450 (2019).

\section{Acknowledgements}

The authors thank Dr. Sabrina Büttner and Dr. Frank Totzke for scientific discussion and Lisa Myketin and Cornelia Schumbrutzki for expert technical assistance. Work included in this study has also been performed in partial fulfillment of the requirements for the doctoral theses of C.W., H.P. and C.K. at the University of Freiburg. This work was supported by the Deutsche Forschungsgemeinschaft (DFG), under Germany's Excellence Strategy (CIBSS - EXC-2189 - Project ID 390939984 to C.M., Cl.K. and F.N.V.), the Heisenberg-Professorship BR 3662/5 (to T.B.), the RTG 2202 and ME 1921/5-1 (to C.M.), the SFB1381 (Project-ID 403222702; to C.M., Cl.K. and F.N.V.), the SFB1177 (Project-ID 259130777; to Cl.K.), project ID 409673687 (to Cl.K.) and the EmmyNoether Programm of the Deutsche Forschungsgemeinschaft (to F.N.V.). Further funding was received from DST-SERB, India (to A.M.), the German Cancer Consortium (DKTK, L627), the Motivate MD college (to H.P.), the Swiss National Science Foundation and the canton of Fribourg (to J.D.), from the European Research Council (ERC) under the European Union's Horizon 2020 research and innovation program under grant agreement No 769065 and from the European Union's Horizon 2020 research and innovation program under grant agreement No 765912 (to Cl.K.). This work reflects only the authors' view and the European Union's Horizon 2020 research and innovation program is not responsible for any use that may be made of the information it contains. Furthermore, this research project was funded by Ministry of Science and Culture of Lower Saxony and Volkswagen Foundation No 762-12-9/19 (ZN3457) (to S.D.).

\section{Author contributions}

C.W., A.M., T.S., V.M., R.E., C.K., P.S.M., Z.H., A.A., S.L., F.A.S., A.SCH., D.P.H., H.P., S.M., S.D. and T.B. performed the experiments. C.W., A.M., T.S., V.M., R.E., A.S., C.K., J.B., J.D., S.D., C.L.K., T.B., F.N.V. and C.M. designed experiments, analyzed and interpreted the data. C.W., A.M., F.N.V. and C.M. developed the project and wrote the manuscript. C.M. coordinated and directed the project. All authors approved the final version of the manuscript.

\section{Funding}

Open Access funding enabled and organized by Projekt DEAL.

\section{Competing interests}

The authors declare no competing interests.

\section{Additional information}

Supplementary information The online version contains supplementary material available at https://doi.org/10.1038/s41467-021-24426-9.

Correspondence and requests for materials should be addressed to F.-N.V. or C.M.

Peer review information Nature Communications thanks Johannes Herrmann and other anonymous reviewers for their contributions to the peer review of this work. Peer review reports are available.

Reprints and permission information is available at http://www.nature.com/reprints

Publisher's note Springer Nature remains neutral with regard to jurisdictional claims in published maps and institutional affiliations.

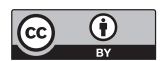

Open Access This article is licensed under a Creative Commons Attribution 4.0 International License, which permits use, sharing, adaptation, distribution and reproduction in any medium or format, as long as you give appropriate credit to the original author(s) and the source, provide a link to the Creative Commons license, and indicate if changes were made. The images or other third party material in this article are included in the article's Creative Commons license, unless indicated otherwise in a credit line to the material. If material is not included in the article's Creative Commons license and your intended use is not permitted by statutory regulation or exceeds the permitted use, you will need to obtain permission directly from the copyright holder. To view a copy of this license, visit http://creativecommons.org/ licenses/by/4.0/.

(C) The Author(s) 2021 